\title{
CCKergic Tufted Cells Differentially Drive Two Anatomically Segregated Inhibitory Circuits in the Mouse Olfactory Bulb
}

\author{
Xicui Sun, ${ }^{1}{ }^{\circledR}$ Xiang Liu, ${ }^{2}$ Eric R. Starr, ${ }^{3}$ and ${ }^{\circledR}$ Shaolin Liu $^{4}$ \\ ${ }^{1}$ Department of Anatomy and Neurobiology, Department of Radiology, University of Maryland School of Medicine, Baltimore, Maryland 21201, \\ ${ }^{2}$ Department of Anatomy and Neurobiology, University of Maryland School of Medicine, Baltimore, Maryland 21201, ${ }^{3}$ Department of Anatomy, \\ Howard University College of Medicine, Washington, DC 20059, and ${ }^{4}$ Department of Anatomy, Howard University College of Medicine, \\ Washington, DC 20059
}

Delineation of functional synaptic connections is fundamental to understanding sensory processing. Olfactory signals are synaptically processed initially in the olfactory bulb $(\mathrm{OB})$ where neural circuits are formed among inhibitory interneurons and the output neurons mitral cells (MCs) and tufted cells (TCs). TCs function in parallel with but differently from MCs and are further classified into multiple subpopulations based on their anatomic and functional heterogeneities. Here, we combined optogenetics with electrophysiology to characterize the synaptic transmission from a subpopulation of TCs, which exclusively express the neuropeptide cholecystokinin (CCK), to two groups of spatially segregated GABAergic interneurons, granule cells (GCs) and glomerular interneurons in mice of both sexes with four major findings. First, CCKergic TCs receive direct input from the olfactory sensory neurons (OSNs). This monosynaptic transmission exhibits high fidelity in response to repetitive OSN input. Second, CCKergic TCs drive GCs through two functionally distinct types of monosynaptic connections: (1) dendrodendritic synapses onto GC distal dendrites via their lateral dendrites in the superficial external plexiform layer (EPL); (2) axodendritic synapses onto GC proximal dendrites via their axon collaterals or terminals in the internal plexiform layer (IPL) on both sides of each bulb. Third, CCKergic TCs monosynaptically excite two subpopulations of inhibitory glomerular interneurons via dendrodendritic synapses. Finally, sniff-like patterned activation of CCKergic TCs induces robust frequency-dependent depression of the dendrodendritic synapses but facilitation of the axodendritic synapses. These results demonstrated important roles of the CCKergic TCs in olfactory processing by orchestrating OB inhibitory activities.

Key words: inhibitory interneuron; neural circuits; olfactory bulb; projection neurons; synaptic plasticity; synaptic transmission

\section{Significance Statement}

Neuronal morphology and organization in the olfactory bulb (OB) have been extensively studied, however, the functional operation of neuronal interactions is not fully understood. We combined optogenetic and electrophysiological approaches to investigate the functional operation of synaptic connections between a specific population of excitatory output neuron and inhibitory interneurons in the OB. We found that these output neurons formed distinct types of synapses with two populations of spatially segregated interneurons. The functional characteristics of these synapses vary significantly depending on the presynaptic compartments so that these output neurons can dynamically rebalance inhibitory feedback or feedforward to other neurons types in the $\mathrm{OB}$ in response to dynamic rhythmic inputs.

\footnotetext{
Received Apr. 1, 2020; revised May 6, 2020; accepted May 21, 2020.

Author contributions: S.L. designed research; X.S., X.L., E.R.S., and S.L. performed research; X.S. and S.L. analyzed data; S.L. wrote the paper.

This work was supported by the National Institute of Deafness and Communication Disorders of the National Institutes of Health Grants R01DC014447. We thank Ms. Corinne A. Perloski for her assistance in animal care and preparation.

$X$. Sun's present address: Department of Diagnostic Radiology, University of Maryland School of Medicine, Baltimore, Maryland 21012.

X. Liu's present address: Department of Otolaryngology, Sun Yat-sen Memorial Hospital, Sun Yat-sen University, Guangzhou, Guangdong 510120, China.
}

\section{Introduction}

Behaving animals actively sample sensory stimuli from their environments (Schroeder et al., 2010). Odorants are presented to olfactory sensory neurons (OSNs) in the olfactory epithelium by

The authors declare no competing financial interests.

Correspondence should be addressed to Shaolin Liu at shaolin.liu@howard.edu.

https://doi.org/10.1523/JNEUROSCI.0769-20.2020

Copyright $\odot 2020$ the authors 
either autonomic respiration or active sniffing in mammals (Wachowiak, 2011). OSNs transduce odor stimuli into electrical signals and transmit them to the olfactory bulb (OB) for processing by neural circuits consisting of the output neurons mitral cells (MCs) and tufted cells (TCs) as well as local inhibitory interneurons (Shepherd, 1972; Shipley and Ennis, 1996).

TCs function in parallel with but differently from MCs (Haberly and Price, 1977; Mori et al., 1983; Orona et al., 1984; Nagayama et al., 2010; Fukunaga et al., 2012; Igarashi et al., 2012; Cavarretta et al., 2018). Unlike MCs with the largest somata arrayed in the compact MC layer (MCL) thus being easily identifiable and extensively studied anatomically and functionally (Cajal, 1911; Price and Powell, 1970a; Macrides and Schneider, 1982; Orona et al., 1984; Lledo et al., 2005), most TCs have somata scattered and intermingled with interneurons in the external plexiform layer (EPL; Macrides and Schneider, 1982) thus impede systematic investigation with traditional physiological approaches. TCs are traditionally classified into three subpopulations: external TCs (ETCs), middle TCs, and internal TCs, with somata in the glomerular layer (GL) and the superficial border of the EPL, the superficial two thirds, and deep one third of the EPL, respectively (Macrides and Schneider, 1982). We recategorized the classical ETCs into two distinct groups, ETCs and superficial TCs (STCs) based on (1) their somata in the GL and superficial one third of the EPL, respectively, and (2) lack of lateral dendrites (ETCs) or with lateral dendrites in the superficial EPL (STCs; Liu et al., 2012). Physiologically, ETCs possess intrinsic properties underlying spontaneous burst firing, while spontaneous firing activities of STCs are synaptically driven (Hayar et al., 2004a; Antal et al., 2006; Liu and Shipley, 2008b). Compared with the extensively studied ETCs (Hayar et al., 2004b; Liu and Shipley, 2008a; De Saint Jan et al., 2009; Gire and Schoppa, 2009; Liu et al., 2013; Whitesell et al., 2013; Vaaga and Westbrook, 2017), the synaptic organization of other TC types is less understood.

TC apical dendrites potentially receive direct input from OSNs and form synapses with local inhibitory interneurons including periglomerular cells (PGCs) and short axon cells (SACs) in the GL (Price and Powell, 1970a; Pinching and Powell, 1971; Parrish-Aungst et al., 2007; Kiyokage et al., 2010; Burton, 2017). Lateral dendrites of TCs form synapses onto distal apical dendrites of GABAergic granule cells (GCs) in the EPL (Rall et al., 1966; Price and Powell, 1970b; Macrides and Schneider, 1982; Mori et al., 1983; Orona et al., 1984), while their axon collaterals terminate in the internal plexiform layer (IPL) and potentially establish synaptic connections with GCs (Price and Powell, 1970b; Haberly and Price, 1977; Kishi et al., 1984; Orona et al., 1984; Schoenfeld et al., 1985; Liu and Shipley, 1994). However, the functional operation of the synapses between TCs and these anatomically segregated interneurons in the $\mathrm{OB}$ has not been systematically investigated.

Here, we addressed these questions by targeting TCs expressing the neuropeptide cholecystokinin (CCK) for three major reasons. First, CCK is expressed predominantly in TCs, especially in the STC type (Seroogy et al., 1985; Liu and Shipley, 1994). Thus, CCK serves as a molecular marker enabling us to selectively label and activate CCKerigc TCs with the optogenetic approach. Second, a recent study demonstrates that CCKergic STCs function distinctively from other STCs (Short and Wachowiak, 2019). Third, CCKergic STCs constitute the intrabulbar associational system (IAS) interconnecting mirror glomeruli (Liu and Shipley, 1994; Belluscio et al., 2002; Lodovichi et al., 2003), where axons of OSNs expressing the same type of odorant receptors terminate (Ressler et al., 1994; Vassar et al., 1994; Mombaerts et al., 1996). Thus, delineating the synaptic organization of the CCKergic TCs would potentially shed light on the functional significance of IAS and mirror glomeruli arrangement in the OB.

\section{Materials and Methods}

Animals

Wild-type male mice (C57BL/6J) and transgenic CCK-Cre mice (Ccktm1.1(cre)Zjh/J, RRID: IMSR_JAX:012706) were obtained from The Jackson Laboratory. The GAD2gfp mice were obtained courtesy of Gabor Szabo (López-Bendito et al., 2004), from the line GAD65_3e/ gfp5.5 \#30 on a genetic background of C57BL6 with backcross to C57BL/6J wild-type mice yielding mice heterozygous for the transgene. TH-GFP mice were courtesy of Kobayashi (Matsushita et al., 2002), generated using a 9.0-kb 5'-flanking region of the $\mathrm{TH}$ gene on a genetic background of C57BL/6J x DBA/2J backcrossed to C57BL/6J yielding mice heterozygous for the transgene. Three additional colonies of transgenic animals were maintained by breeding homozygous CCK-Cre male with wild-type C57BL/6J (CCK-Cre heterozygous), GAD65-GFP heterozygous (CCK-Cre x GAD65-GFP), or TH-GFP heterozygous (CCK-Cre $\mathrm{x}$ TH-GFP) female mice, respectively. Animals were maintained with a standard 12/12 h light/dark cycle and given food and water ad libitum. All experimental procedures were performed in accordance with protocols approved by the Institutional Animal Care and Use Committees (IACUCs) of University of Maryland at Baltimore and Howard University.

\section{Channelrhodopsin 2 (ChR2) expression}

Virus injection was performed as previously described (Liu et al., 2013). Briefly, the adeno-associated virus serotype 5 (AAV2.5) carrying fusion genes for ChR2 and enhanced yellow fluorescent protein (EYFP; AAV-EF1a-double floxed-hChR2(H134R)-EYFP) or mCherry (AAV-EF1a-double floxed-hChR2(H134R)-mCherry; Tsai et al., 2009; University of Pennsylvania Vector Core or Addgene) was injected into the superficial EPL on the medial side of each OB of animals between their fourth and sixth postnatal week. Under deep anesthesia, skull was exposed, and a craniotomy (1 $\mathrm{mm}$ in diameter) was drilled on the midsagittal line between two OBs with a typical coordinate at $3.95 \mathrm{~mm}$ from bregma. AAV2.5 was injected into two points within the superficial EPL on the medial side of each bulb $(0.3 \mathrm{~mm}$ from the midline, depth of 1.7 , and $1.2 \mathrm{~mm})$ at a rate of $0.6 \mu \mathrm{l} / \mathrm{min}$ for $30 \mathrm{~s}$ via a nanoliter injector (Nanoject III, Drummond Scientific). At three to four weeks after virus injection, acute horizontal OB slices were prepared for experiments.

\section{Slice preparation}

Acute OB slices were prepared from 9- to 10-week-old male or female mice as described previously (Liu and Shipley, 2008b). Briefly, animals were deeply anesthetized with isoflurane using the IACUC-approved drop method before decapitation. Then OBs were rapidly dissected and mounted onto the specimen disk of a VT1200S vibratome using superglue. Horizontal $\mathrm{OB}$ slices $(350 \mu \mathrm{m})$ were cut in an ice-cold and oxygenated (95\% $\mathrm{O}_{2}-5 \% \mathrm{CO}_{2}$ ) sucrose-based artificial CSF (sucrose-ACSF) containing the following: $210 \mathrm{~mm}$ sucrose, $2.5 \mathrm{~mm} \mathrm{KCl}, 1.2 \mathrm{~mm}$ $\mathrm{NaH}_{2} \mathrm{PO}_{4}, 2.6 \mathrm{~mm} \mathrm{MgSO}_{4}, 0.5 \mathrm{~mm} \mathrm{CaCl}_{2}, 26 \mathrm{~mm} \mathrm{NaHCO}_{3}$, and $10 \mathrm{~mm}$ glucose. After $30 \mathrm{~min}$ of incubation in normal ACSF at $30^{\circ} \mathrm{C}$, slices were then transferred to ACSF at room temperature (RT) until they were used for recordings. Normal ACSF was continuously bubbled with $95 \%$ $\mathrm{O}_{2}-5 \% \mathrm{CO}_{2}$ and had the following composition: $124 \mathrm{~mm} \mathrm{NaCl}, 2.5 \mathrm{~mm}$ $\mathrm{KCl}, 1.25 \mathrm{~mm} \mathrm{NaH} \mathrm{PO}_{4}, 2.0 \mathrm{mM} \mathrm{MgSO}_{4}, 2.0 \mathrm{mM} \mathrm{CaCl}_{2}, 26 \mathrm{~mm}$ $\mathrm{NaHCO}_{3}$, and $10 \mathrm{~mm}$ glucose. During experiments, slices were perfused at $3 \mathrm{ml} / \mathrm{min}$ with ACSF equilibrated with $95 \% \mathrm{O}_{2}-5 \% \mathrm{CO}_{2}$ and warmed to $30^{\circ} \mathrm{C}$.

\section{Electrophysiology}

Whole-cell patch-clamp recordings were made from OB neurons visualized using BX50WI (Olympus) or Axio Examiner (Zeiss) fixed-stage 
upright epifluorescence microscope equipped with near-infrared differential interference contrast (DIC) optics. SACs and PGCs were identified by their expression of TH-GFP and GAD65-EGFP, respectively.

For post hoc reconstruction of GCs, biocytin $(0.2 \%)$ was included in the internal solution. Current or voltage signals were recorded with a MultiClamp 700B amplifier (Molecular Devices) and lowpass filtered at $4 \mathrm{kHz}$ and sampled at $10 \mathrm{kHz}$ with a DIGIDATA 1322A or 1550B 16-bit analog-to-digital converter (Molecular Devices) using Clampex 10.3 or 11.1 (Molecular Devices). Patch recording electrodes were pulled from standard-wall glass capillary tubes without filament (Sutter Instrument). Patch pipettes (4-7 $\mathrm{M} \Omega$ ) for whole-cell recording and contained the following: $115 \mathrm{~mm}$ K-gluconate, $5.0 \mathrm{~mm}$ EGTA, $0.63 \mathrm{~mm} \mathrm{CaCl}_{2}, 5.5 \mathrm{~mm} \mathrm{MgCl}_{2}, 10 \mathrm{~mm}$ HEPES, $3 \mathrm{~mm} \mathrm{Na}_{2}$-ATP, $0.3 \mathrm{~mm} \mathrm{Na}_{3}$-GTP, and $14 \mathrm{~mm}$ Tris-phos-

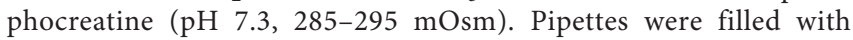
ACSF for cell-attached recordings. Tetrodotoxin (TTX; $1 \mu \mathrm{M}$ ) was bath-applied to eliminate action potential-dependent circuit effects.

\section{Immunochemical staining}

OB slices with $0.2 \%$ (w/v) biocytin-filled GCs were immediately kept in $4 \%$ paraformaldehyde (PFA) at $4^{\circ} \mathrm{C}$ overnight. After three $(5 \mathrm{~min}$ each) washes with $0.05 \mathrm{M}$ PBS, slices were incubated in a blocker solution on a shaker for $1 \mathrm{~h}$. Blocker solution was made by 0.05 м PBS with the addition of bovine serum albumin (BSA) and Triton X-100 at final concentrations of $1 \%(\mathrm{w} / \mathrm{v})$ and $0.5 \%(\mathrm{v} / \mathrm{v})$, respectively. The slices were then transferred to and kept in this blocker solution containing streptavidin-Cy3 $(1 \mu \mathrm{g} / \mathrm{ml})$ covered with aluminum foil to prevent light exposure at RT on a shaker for $7 \mathrm{~h}$. Following three ( 5 min each) rinses with $0.05 \mathrm{M}$ PBS to terminate streptavidin-Cy3 staining, slices were treated with 0.05 M PBS containing 4',6-diamidino-2-phenylindole (DAPI; $5 \mu \mathrm{g} / \mathrm{ml}$ ) at RT in the dark for $10 \mathrm{~min}$. DAPI staining was terminated by three ( 5 min each) washes with PBS before slices were wet mounted and cover-slipped with fluorescence mounting media. Biocytin-filled cells with $\mathrm{Cy} 3$ staining were scanned and reconstructed under a confocal microscope.

For CCK protein staining, CCK-Cre mice were injected intracerebroventricularly with $10 \mu \mathrm{g}$ colchicine dissolved in $5 \mu \mathrm{l}$ of saline at four weeks after receiving AAV virus injection in their OBs. Forty-eight hours later, animals were anesthetized and transcardially perfused with saline followed by $4 \%$ PFA before OBs were dissected out and kept in $4 \%$ PFA at $4^{\circ} \mathrm{C}$ for $24 \mathrm{~h}$. OBs were then cut into $30-\mu \mathrm{m}$ sections with a vibratome (Leica VT1000s) in 0.05 m PBS. OB sections were rinsed with $0.05 \mathrm{M}$ PBS for three times ( $5 \mathrm{~min}$ each) before being incubated in blocking solution: 0.05 м PBS containing $0.5 \%$ Triton X-100 and $1 \%$ BSA. One hour later, sections were incubated with rabbit polyclonal anti-CCK8 antibody (1:1000; C2581; Sigma-Aldrich) in blocking solution for $48 \mathrm{~h}$ at $4^{\circ} \mathrm{C}$. Following three times ( $5 \mathrm{~min}$ each) of rinse with PBS, tissue sections were treated by blocking solution containing Alexa Fluor 488-conjugated goat anti-rabbit antibody (1:1000; A32731; ThermoFisher Scientific) in dark for $2 \mathrm{~h}$ at RT. After termination of secondary antibody reaction by three times ( 5 min each) of rinse with PBS, sections were incubated in PBS containing $5 \mu \mathrm{g} / \mathrm{ml}$ DAPI at RT for $10 \mathrm{~min}$. Finally, after being rinsed with PBS for three times ( 5 min each) to terminate DAPI staining, sections were mounted with anti-fade fluorescence mounting medium and coverslipped for confocal imaging.

\section{Electrical and optical stimulation}

Electrical stimulation was delivered by bipolar glass electrodes made from theta borosilicate tubes (Sutter Instrument). The isolated and constant current stimulation pulses $(100 \mu \mathrm{s})$ were triggered by a Master- 8 or Master-9 stimulator with a stimulus isolator ISO-FLEX (AMPI). Optical stimulation was produced by a $100 \mathrm{~mW}, 473 \mathrm{~nm}$, diode-pumped, solidstate laser MBL-III-473 (Optoengine) and gated with a laser shutter LST200 (NMLaser Products). Optical stimuli were delivered from a multimode optical fiber (ThorLabs) with opening diameter of $25 \mu \mathrm{m}$ ( $0.1 \mathrm{NA}, \sim 7^{\circ}$ beam divergence). Laser delivered with this type of fiber optic produced an elliptical illumination area on brain slices in the recording chamber with typical major and minor diameters of 60 and
$40 \mu \mathrm{m}$, respectively. Optical power delivered at the fiber tip was calibrated with a PM20A power meter (ThorLabs). Onset and duration of optical stimulation were measured during every experiment by splitting $1 \%$ of the laser beam out to a high-speed (30-ns rise time) silicon photosensor (model $818-\mathrm{BB}$, Newport) and recorded by the same MultiClamp 700B amplifier.

\section{Data analysis and statistical tests}

All numerical data were presented as mean \pm SEM. Action potentials were detected in Clampfit 10.7 using a detection threshold of $-40 \mathrm{mV}$ to exclude EPSP detection or a detection threshold of $-20 \mathrm{pA}$ relative to baseline for cell-attached data. Group data graphing or plotting was performed with Origin Pro 2020 (Origin Lab). Statistical details of experiments are listed in Results. Statistical significance of population responses was determined by using paired $t$ test for comparing data examining NBQX and APV effects on the same group of cells (Fig. $4 D-$ $F$ ), one-way ANOVA test for linear regression data (Figs. $2 F, 6 F, G, 7 F$, $G$ ), or ANOVA one-way repeated measure (ANOVAOneWayRM) with Bonferroni post hoc comparisons for multiple $(>2)$ measurements of the same group of cells with paired comparisons (Figs. 5F, 6E, 7E, 8D,E, 9G, $H, 10 D-G, 11 E, F)$ in Origin Pro 2020. Statistical significance was set at $p<0.05$ for all tests.

\section{Drugs delivery and chemicals}

Drugs were all bath applied. DL-2-amino-5-phosphonopentanoic acid sodium salt (APV; $50 \mu \mathrm{M}$ ), 2,3-dioxo-6-nitro-1,2,3,4-tetrahydrobenzo[f]quinoxaline-7-sulfonamide disodium salt (NBQX disodium salt; $10 \mu \mathrm{M}$ ), gabazine (SR95531, $10 \mu \mathrm{M}$ ), and octahydro-12-(hydroxymethyl)-2-imino5,9:7,10a-dimethano-10aH-[1,3] dioxocino[6,5-d]pyrimidine-4,7,10,11,12pentol citrate (TTX citrate; $1 \mu \mathrm{M}$ ) were purchased from Tocris Cookson. Cy3 streptavidin and Alexa Fluor 488-conjugated goat anti-rabbit antibody were from ThermoFisher Scientific. All other chemicals were purchased from Sigma-Aldrich. All drugs were dissolved in distilled water as stock solution and diluted 1000 times with ACSF to final concentrations.

\section{Results}

\section{Selective labeling and activation of CCKergic STCs}

To characterize CCKergic TC responses to ON input, we adopted the optogenetic approach by injecting Cre-dependent AAV2.5-ChR2-EYFP into the OB of CCK-Cre mice to selectively label these excitatory projection neurons. As shown in Figure $1 A$, left panel, virus injection into the $\mathrm{OB}$ induced consistent EYFP expression predominantly located in the superficial EPL, GL, and IPL, which correspond to locations of somata, apical dendrite tufts, and axons of the CCK-containing and IAS-forming TCs, respectively (Haberly and Price, 1977; Kishi et al., 1984; Schoenfeld et al., 1985; Seroogy et al., 1985; Liu and Shipley, 1994). Somatic counting analysis (Fig. 1A, right panel) showed that vast majority (84.6\%) of EYFP-expressing cells had somata located in the superficial (126/150 cells in three mice) one third rather than middle (9/150 cells) or deep (15/150 cells) one third of the EPL (Fig. 1A, right panel, $F$ ), suggesting that our optogenetic approach preferentially labels CCKergic STCs. Furthermore, immunohistochemical staining revealed that $88.9 \pm 1.7 \% \quad(n=127$ cells from three mice) of $\mathrm{EYFP}^{+}$cells in the EPL were CCK protein-positive (Fig. 1B). Since the axonal transporter blocker colchicine is required for detection of CCK peptide in cell somata, the remaining $\sim 11 \%$ may reflect TCs with undetectable level of CCK protein in cell bodies at the given dose of colchicine intracerebroventricular injection. Consistent with TC morphology (Macrides and Schneider, 1982; Mori et al., 1983; Orona et al., 1984; Schoenfeld et al., 1985; Liu and Shipley, 1994), the vast majority (91/100 cells in three mice) of the EYFP-expressing cells showed 

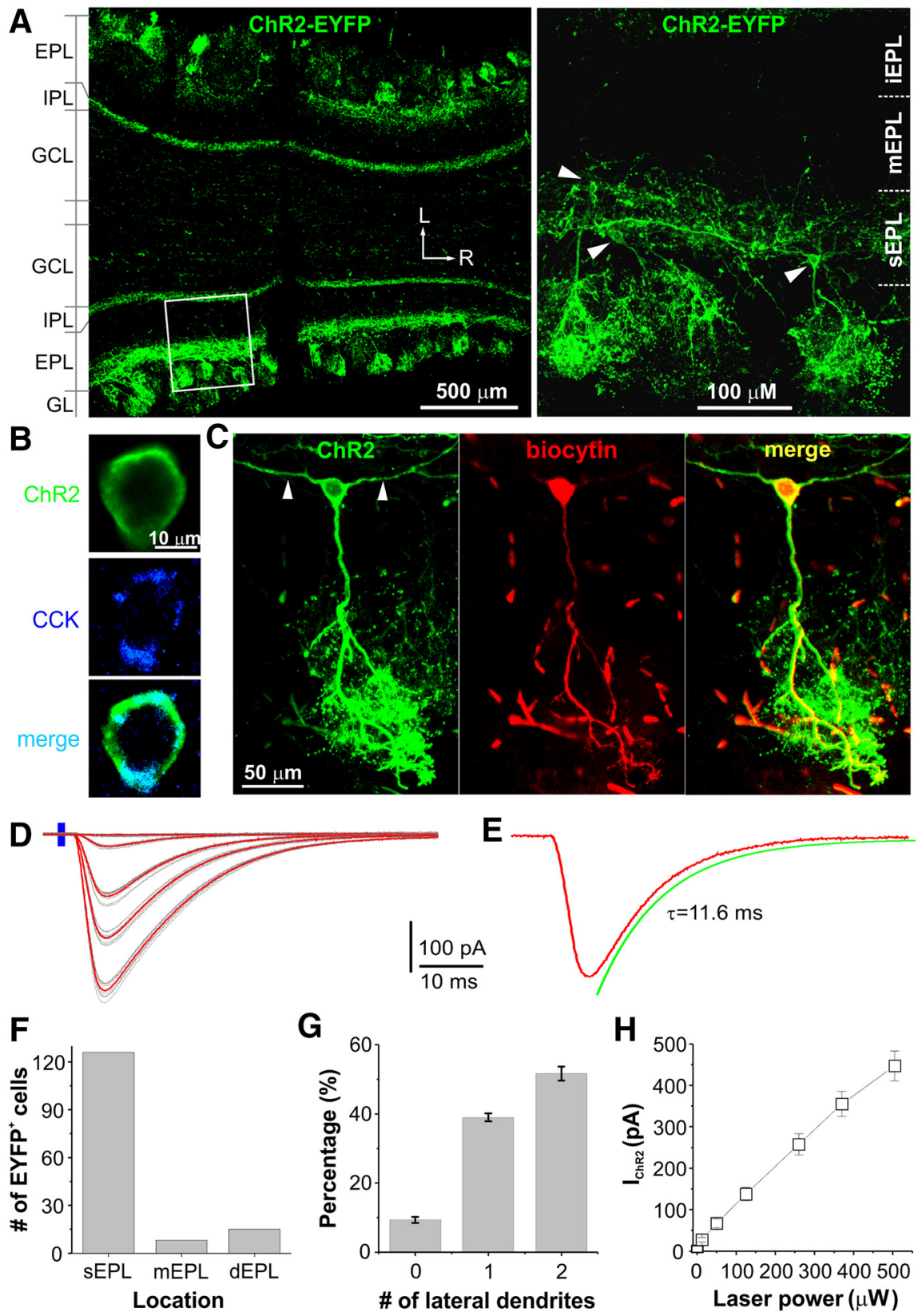

Figure 1. Selective labeling and activation of CCKergic TCs in the olfactory bulb. A, left panel, Confocal image of a horizontal OB section showing expression of ChR2-EYFP. Right panel, Blown-up from left panel showing EYFP-expressing TC somata and division of the EPL into three equivalent portions: superficial one third (sEPL), middle one third (mEPL), and internal one third (iEPL). Arrow heads point to somata. L: lateral, R: rostral. B, Confocal images showing the colocalization of ChR2-EYFP (green, top) and CCK protein (blue, middle) in TCs. C, One recorded STC labeled with two markers ChR2-EYFP (green) and biocytin (red) filled via a patch electrode with lateral dendrites highlighted by arrowheads. D, Typical ChR2 current traces evoked by 2-ms laser blue light (vertical blue bar) at different power levels. Gray: individual traces, red: averaged traces for each laser power level. $\boldsymbol{E}$, Typical averaged trace showing the ChR2 current decay time constant measurement. $\boldsymbol{F}, \boldsymbol{G}$, Bar graphs showing the number of EYFP ${ }^{+}$cells distributed in each of three portions of the EPL and percentage of EYFP ${ }^{+}$cells with different number of lateral dendrites. $\boldsymbol{H}$, Plot showing the relationship of the averaged ChR2 current peak amplitude ( $n=7$ cells) with laser power. GCL: granule cell layer.

one (39/100 cells) or two (52/100 cells) lateral dendrites (Fig. 1C, $G)$ but all had single apical dendrites confined to individual glomeruli (100/100 cells; Fig. 1C). The rest 9\% (9/100 cells) with no lateral dendrites but having somata residing in the boundary region between GL and EPL may represent STCs with lateral dendrites truncated during slicing or ETCs, which have no lateral dendrites (Hayar et al., 2004a,b; Liu and Shipley, 2008b). Collectively, these findings demonstrated that our optogenetic approach enabled us to preferentially label CCKergic STCs in the OB. Thus, our subsequent physiological studies mainly focused on this subpopulation of TCs.
To characterize the ChR2-mediated current or excitation in the CCKergic STCs, the selective blockers of AMPA (NBQX, 10 $\mu \mathrm{M})$ and NMDA (D-APV, $50 \mu \mathrm{M}$ ) receptors as well as $\mathrm{GABA}_{\mathrm{A}}$ receptors (gabazine/GBZ, $10 \mu \mathrm{M}$ ) were bath-applied throughout the experiments to minimize circuit influence. In these conditions, brief $(2 \mathrm{~ms})$ blue laser light $(473 \mathrm{~nm})$ evoked intensity-dependent inward currents in the EYFP-expressing cells voltage clamped at $-60 \mathrm{mV}$ (Fig. $1 D, H$ ). The decay time constant of this inward current was $11.9 \pm 0.9 \mathrm{~ms}$ ( $n=7$ cells; Fig. $1 E)$, which is consistent with the kinetics of the humanized ChR2-mediated current (Lin et al., 2009). To ensure activation consistency and 
A

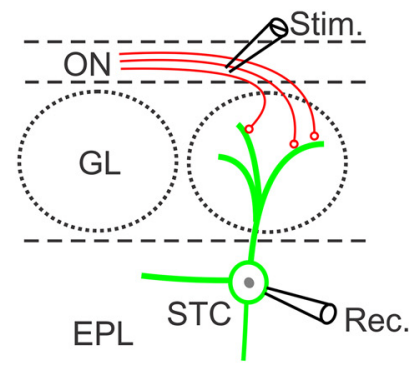

B
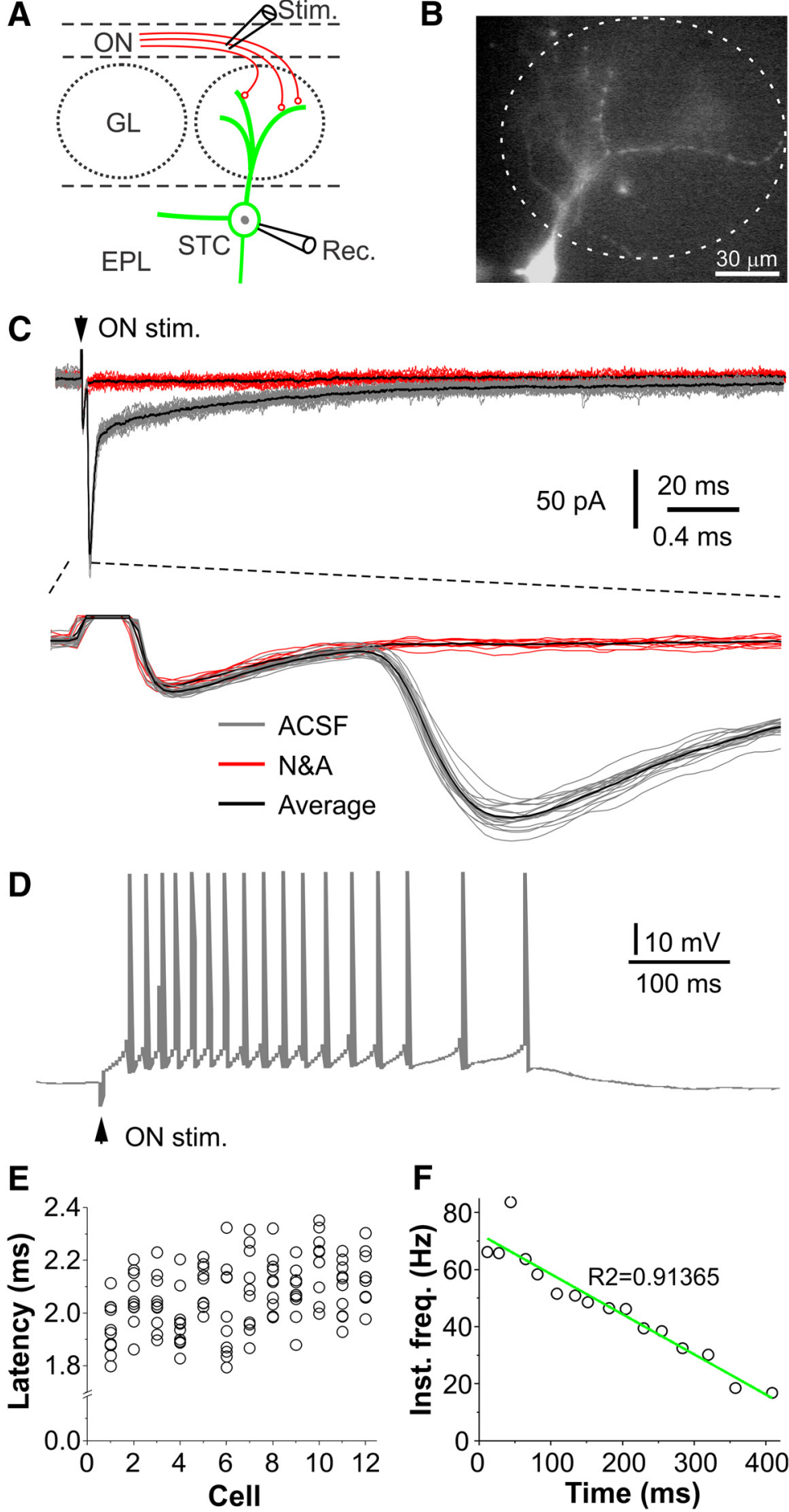

Figure 2. CCKergic STCs receive monosynaptic input from the ON. $\boldsymbol{A}$, Schematic showing experimental design. $\boldsymbol{B}$, Epifluorescence microscopic photograph showing a recorded EYFP ${ }^{+}$STC. C, Top, typical traces showing the 0N stimulation (ON stim., arrowhead)-evoked EPSCs in a STC in OB slice perfused with ACSF (gray traces) or after addition of the fast glutamatergic receptor blockers NBQX and APV (N\&A, red traces). Bottom, Blown-up from the top traces showing the consistent EPSC onset latencies. Black traces represent the average. D, Typical trace showing ON stimulation-evoked spike response in the same STC shown in C. $\boldsymbol{E}$, Symbol plot showing onset latencies of ON-evoked EPSCS in 12 STCS. $\boldsymbol{F}$, Fitting plot showing the negative correlation of the instant frequency of the burst spike response with time of the trace shown in $\boldsymbol{D}$.

data comparability, we used the same level of laser power $(260 \mu \mathrm{W})$ for all subsequent experiments. Altogether, our results demonstrate the effectiveness of our optogenetic approach to selectively label and activate the CCKergic STCs in the $\mathrm{OB}$.

\section{CCKergic STCs receive direct input from the olfactory nerve $(\mathrm{ON})$}

A recent calcium-imaging study demonstrated that CCKergic STCs responded to odor stimulation with consistently shorter onset latencies than MCs and other nonCCKergic STCs, indicating the CCKergic STCs function as a distinct subpopulation of TCs receiving direct and faithful OSN input (Short and Wachowiak, 2019). To test this, we made whole-cell recordings from the EYFP-expressing STCs (Fig. 2A, $B$ ) in $\mathrm{OB}$ slices in both voltage and current clamp. As shown by Figure $2 C$, electrical ON stimulation evoked EPSCs in STCs voltage clamped at $-60 \mathrm{mV}$. These $\mathrm{ON}$ evoked EPSCs were completely abolished by the fast glutamatergic receptor blockers NBQX and APV (Fig. 2C, red traces compared with gray traces) and exhibited consistently short onset latencies $(2.1 \pm$ $0.02 \mathrm{~ms}, n=12)$ with a jitter of $112.4 \pm$ $0.01 \mu \mathrm{s}(n=12$; Fig. $2 C$, bottom trace, $2 E)$, supporting their nature of glutamatemediated EPSCs. In current clamp, STCs responded to electrical $\mathrm{ON}$ stimulation with a burst of action potentials (Fig. 2D), duration of which varies from cell to cell ranging from 297.6 to $497.9 \mathrm{~ms}$ ( $452.6 \pm$ $11.3 \mathrm{~ms}, n=6$ ). The average number of action potentials in each burst response was $21.3 \pm 4.8 \quad(n=6)$. Each ON-evoked response exhibited a spike frequency adaptation, a progressive reduction in the instant spike frequency following an initial increase (Fig. 2D,F). This may reflect the kinetic profiles of the underlying ON-elicited EPSCs, i.e., an initial brief large component followed by a prolonged small and gradually decaying component (Fig. 2C), or intrinsic properties of STCs.

Taken together, these results substantiate that CCKergic STCs respond to the glutamate-mediated, monosynaptic ON input with bursts of action potentials.

\section{STCs provide direct synaptic input to GCs}

Previous ultrastructural evidence supports synaptic connections between the CCKergic TCs and GCs in the IPL (Price and Powell, 1970b; Kishi et al., 1984; Orona et al., 1984; Liu and Shipley, 2008b). However, the functional operation of these synapses remains elusive. This could be due to the lack of experimental approaches to selectively activate TCs. GCs are broadly classified into two major populations: superficial and deep GCs. The superficial GCs have somata residing in the superficial portion of the GC layer and apical dendrites reaching the superficial EPL where they potentially form synapses with TC lateral dendrites while deep GCs have somata in the deep 
portion of the GC layer and apical dendrites confined to the deep EPL region (Price and Powell, 1970b; Orona et al., 1983). Although apical dendrites of all GCs traverse the IPL where they potentially form axodendritic synapses with TC axon collaterals, a previous study suggests only the superficial type of GCs synapse with TC axon collaterals in the IPL (Schoenfeld et al., 1985). Thus, TCs potentially form both dendrodendritic and axodendritic synapses via their lateral dendrites and axon collaterals in the superficial EPL and IPL with distal and proximal dendrites of the same group of GCs, respectively. Thus, we chose to record from superficial GCs to test these possibilities.

In order to optogenetically activate CCKergic TCs and selectively record from GCs, we crossbred CCK-Cre mice with GAD65-GFP mice to generate a CCK-Cre x GAD65-GFP mouse line that enabled us to selectively express ChR2 in the CCKergic TCs and identify the GFP-tagged GABAergic GCs that express GAD65 (ParrishAungst et al., 2007), a key enzyme for biosynthesis of GABA. As shown by Figure 3, at four weeks after AAV-ChR2-mCherry injection into the medial side of the OB of CCK-Cre x GAD65GFP mice, intense expression of mCherry-ChR2 was confined to the superficial EPL, GL, and the IPL, corresponding to the locations of somata, apical dendritic tufts, and axons of STCs, suggesting predominant ChR2mCherry expression in the STCs. Meanwhile, numerous GAD65-GFP-expressing cells were present in the GC layer thus enabled us to selectively record from GABAergic GCs, majority of which express GAD65 (Parrish-Aungst et al., 2007).

With this approach, we first investigated whether superficial GCs received synaptic input from CCKergic TC lateral dendrites. Whole-cell voltage-clamp recordings were performed from the GAD65-GFP-expressing cells in the superficial half of the GC layer, whereas optical stimulation was delivered to the superficial EPL to target CCKergic STC soma and lateral dendrites (Fig. 4A). The stimulation site of the superficial EPL had a typical distance of $150 \mu \mathrm{m}$ from the IPL and the laser-delivering fiber optic was oriented to facing toward the GL such that light spread to the deep lamina was minimized to avoid confounding responses. Biocytin (0.2\%) was included in the recording pipette solution for post hoc reconstruction, which verified the cellular identity of the recorded GCs (Fig. 4B,C). Under these conditions, we found that two paired optic stimuli (2-ms duration each, 100-ms interval) delivered to the superficial EPL region with a radial angle perpendicular to the mCherryexpressing STC axon-compacted IPL right above the recorded cells (Fig. 4B,C). To achieve maximal responses, the optical stimulation was move back and forth in a directions parallel to the IPL right above the recorded cells. Recordings were continued only in GCs that responded to optical stimulation of the superficial EPL since the nonresponsive ones could likely be those with apical dendrites being truncated during slice preparation. As shown in Figure $4 D$, top (gray) traces, the paired stimuli evoked paired inward currents in GCs voltage clamped at $-60 \mathrm{mV}$. These inward currents were completely eliminated by bath application of $10 \mu \mathrm{M} \mathrm{NBQX}$ and $50 \mu \mathrm{M}$ D-APV (Fig. 4D, top blue traces) in all nine recorded cells, supporting their nature of glutamate-mediated EPSCs. These superficial EPL-evoked EPSCs exhibited consistently short onset latencies $(2.1 \pm 0.1 \mathrm{~ms}, n=9)$ with an averaged synaptic jitter (latency deviation) of $392.4 \pm 26$ $\mu$ s ( $n=9$; Fig. $4 D$, bottom left traces). In each of the same group of GCs, we moved the optic fiber toward the cell body direction for $\sim 150 \mu \mathrm{m}$ to stimulate the mCherry-expressing IPL right above the recorded cells whereas opening of the fiber optic was reoriented to face away from the EPL thus precluding activation 
A

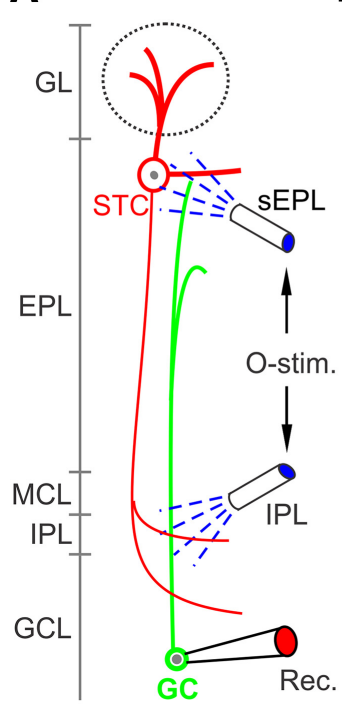

B

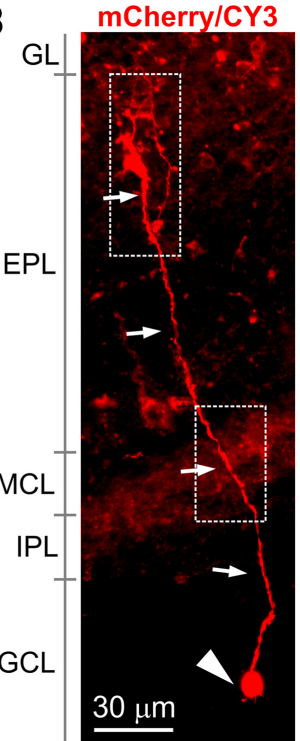

EYFP

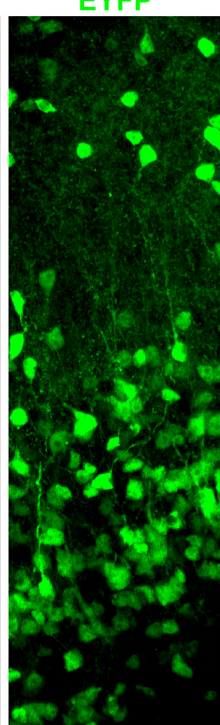

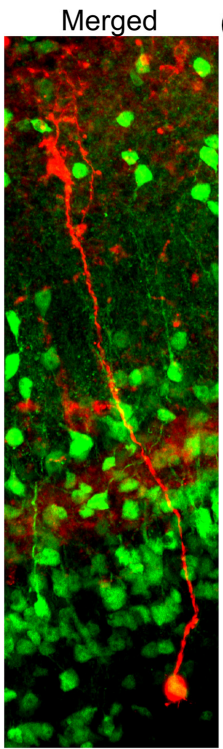

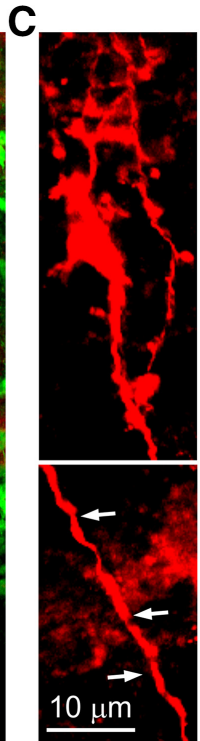

D

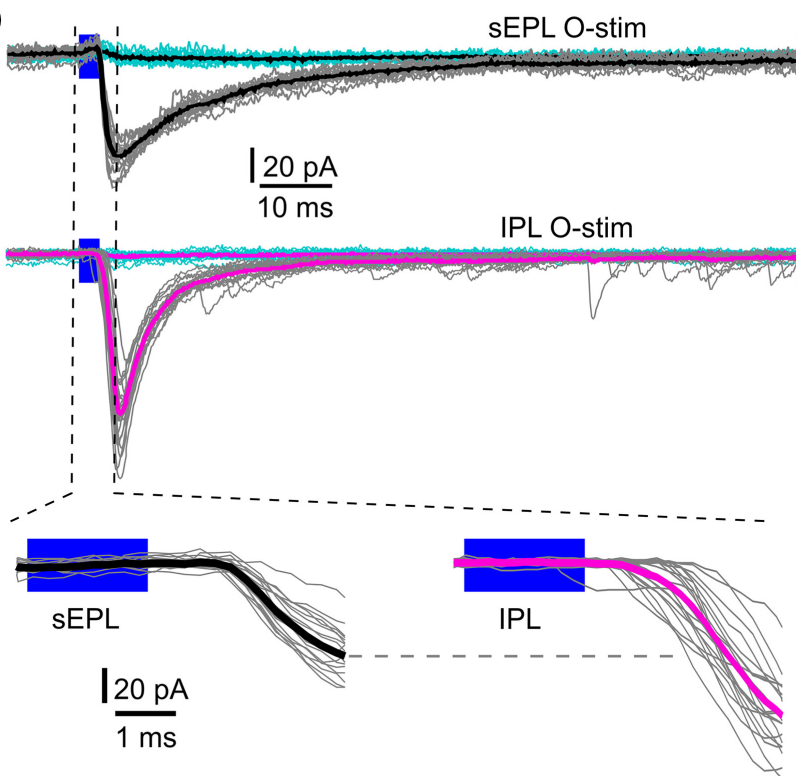

E

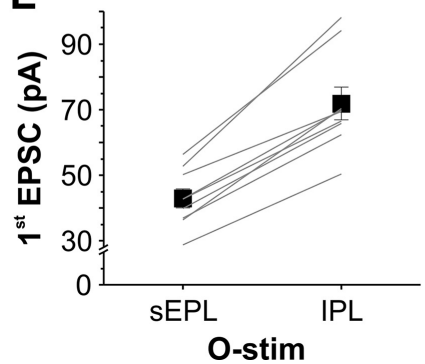

F

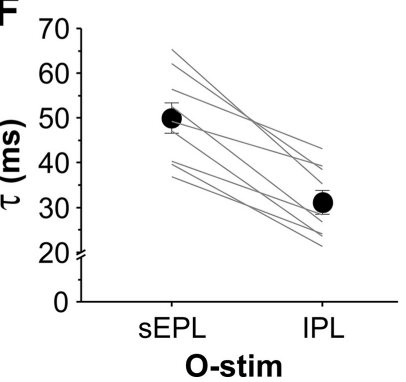

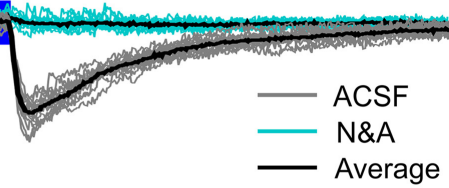

Average
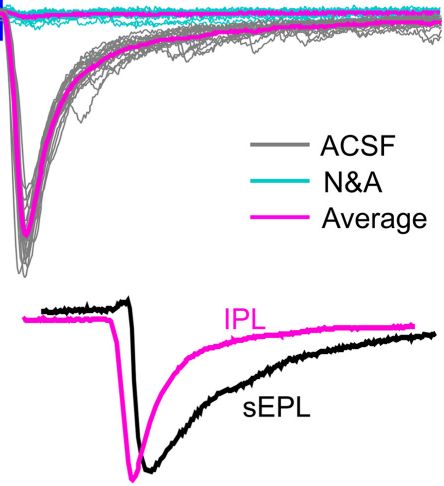

Scaled

G

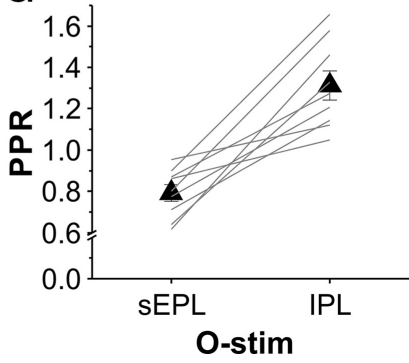

Figure 4. CCKergic TCs drive GCs via both lateral dendrites and axons. A, Schematic showing experimental design. B, Confocal images showing ChR2-mCherry expression in TC axons in the IPL and somata and lateral dendrites in the superficial EPL, a biocytin-filled and Cy3-tagged GC (red, left), GAD65-GFP-expressing interneurons (middle), and merged image (right). $\boldsymbol{C}$, Blown-up images of the areas labeled in the left panel in $\boldsymbol{B}$ showing the $\mathrm{GC}$ apical dendritic tuft (top) and the relationship between the $\mathrm{GC}$ proximal dendrite (arrowheads) and the cross-sectioned mCherry-expressing TC axon fibers. D, Top and middle, typical voltage-clamp traces showing EPSCS recorded in the same GC shown in $\boldsymbol{B}$ evoked by paired-pulse optical stimulation (0-stim.) delivered to the superficial EPL (sEPL, top traces) radially above or the IPL (middle traces) right superficial to the recorded cell as shown in $\boldsymbol{A}$. Gray and cyan traces were recorded in ACSF and in the presence of NBQX and APV (N\&A), respectively, whereas black and magenta traces represent the average for each condition. Bottom, left and middle, Blown-up from the top and middle traces, respectively; right traces are blown-up of the averaged first EPSCs shown in the top and middle traces to highlight the difference in EPSC decay kinetics. $\boldsymbol{E}-\boldsymbol{G}$, Plots showing the amplitude $(\boldsymbol{E})$ and decay time constant $(\boldsymbol{F})$ of the first EPSC, and PPR (EPSC2/EPSC1; G) of EPSCs evoked by the paired-pulse optical stimulation of the sEPL or IPL in seven GCS. 
A

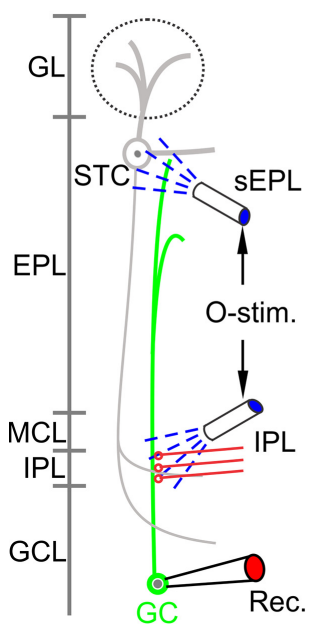

B

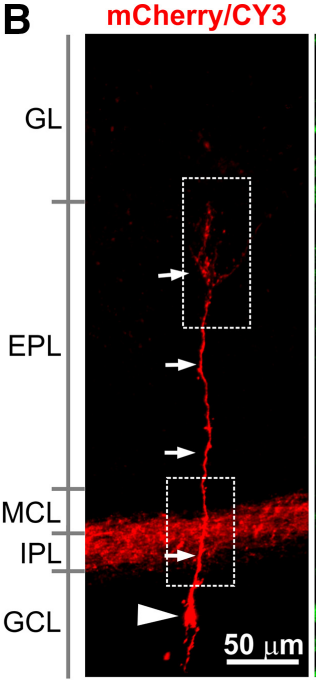

EYFP

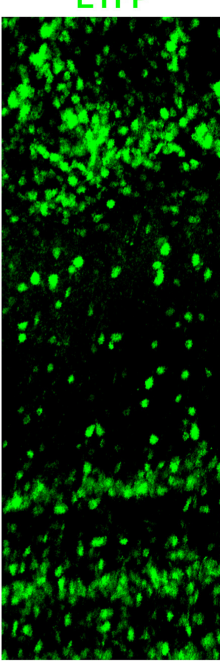

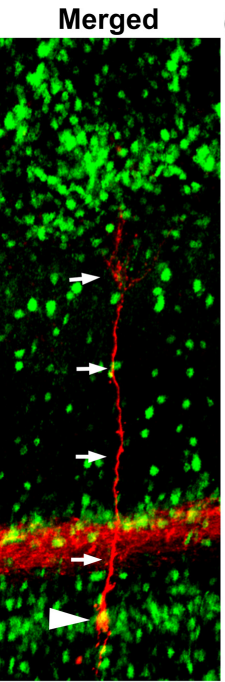

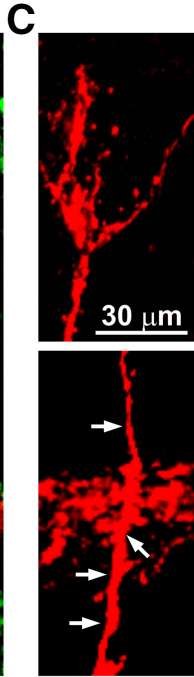

D
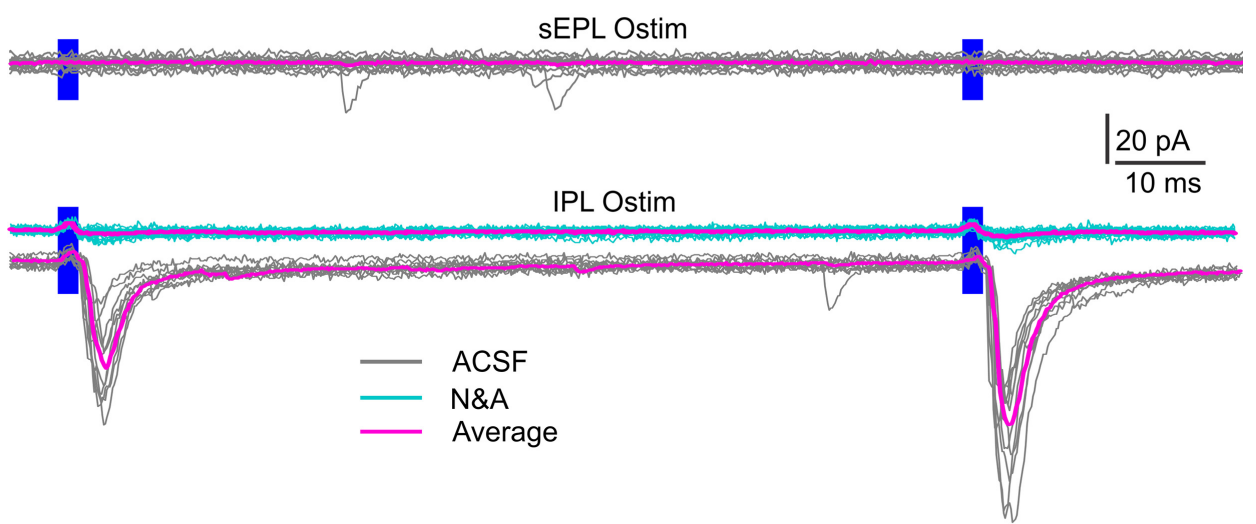

E

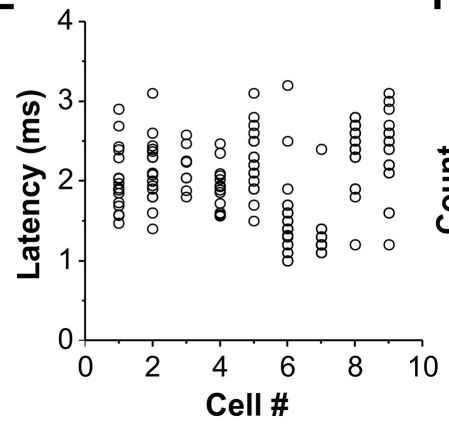

F

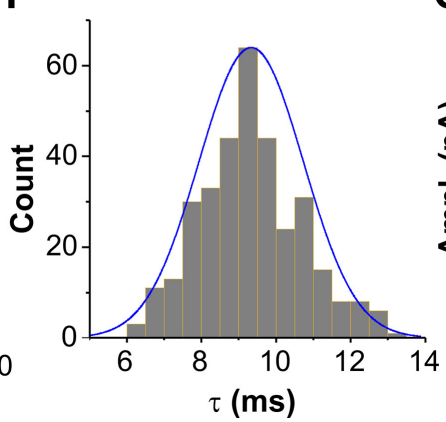

G

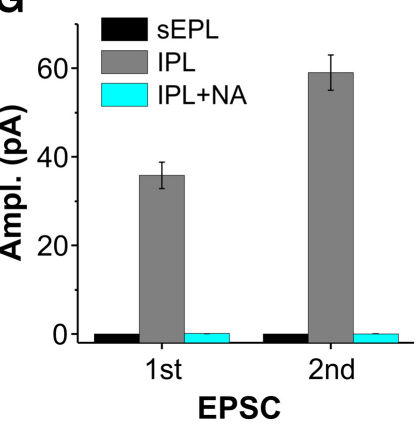

Figure 5. CCKergic TCs excite GCs via axons in the IPL on the opposite side of the same bulb. $\boldsymbol{A}$, Schematic showing experimental design. $\boldsymbol{B}$, Confocal images showing ChR2-mCherry expression in TC axons in the IPL on the opposite side of the bulb and a biocytin-filled and Cy3-tagged GC (red, left), GAD65-GFP-expressing interneurons (middle), and the merged image (right). $C$, Blown-up images of the areas labeled in the left panel in $\boldsymbol{B}$ highlighting the $\mathrm{GC}$ apical dendritic tuft (top) and the relationship between the $\mathrm{GC}$ proximal dendrite (arrowheads) and the crosssectioned mCherry-expressing TC axon fibers. $\boldsymbol{D}$, top and bottom, Typical voltage-clamp traces showing responses recorded in the same GC shown in $\boldsymbol{B}$ evoked by paired-pulse optical stimulation delivered to the superficial EPL (sEPL; top traces) radially above or the IPL (bottom traces) right above the recorded cell as shown in $A$. Gray and cyan traces were recorded in ACSF and in the presence of NBQX and APV (N\&A), respectively, whereas magenta traces represent the average for each conditions. $\boldsymbol{E}$, Symbol plot showing onset latencies of the IPL-evoked individual EPSCS in nine GCS. F, Histogram showing the distribution of the decay time constant data collected from nine GCS. G, Bar graph showing the average amplitude of EPSCs in nine GCS evoked by paired optical stimuli delivered to the sEPL or IPL in ACSF or in the presence of NBQX and APV (IPL +N\&A).

of cellular components in the EPL (Fig. 4A). In these conditions, the identical paired optical stimuli elicited two paired inward currents in all tested cells (Fig. $4 D$, middle gray traces). Similarly, these inward currents were completely blocked by bath applied NBQX and APV (Fig. 4D, middle blue traces), verifying their EPSC nature. Like the superficial EPL-evoked EPSCs, these IPLelicited EPSCs had short onset latencies $(2.1 \pm 0.2 \mathrm{~ms}, n=9)$ with an averaged synaptic jitter of and $245.2 \pm 52.4 \mu$ s (Fig. $4 D$, bottom middle traces). The relatively high jitters of these EPSCs may reflect the different level of ChR2 expression in the lateral dendrites and axons among TCs or differences in the ChR2 activation level because these cellular components situated in various depth from the tissue surface thus were exposed to the laser light of variant intensities. Thus, the short onset latencies and unobservable failure of GC responses to paired-pulse stimulation suggest a monosynaptic transmission from the CCKergic TC lateral dendrites and axons to GCs (Miles, 1986; Yoshimura and Jessell, 1989; Gil et al., 1999; Sabatini and Regehr, 1999; Doyle and 
A

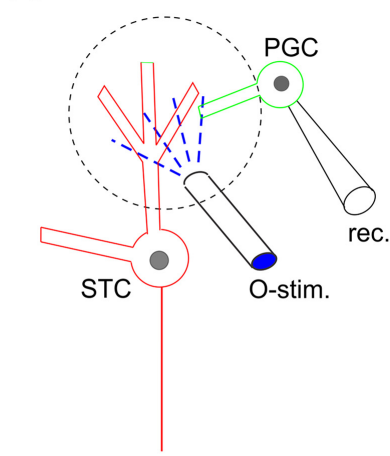

B

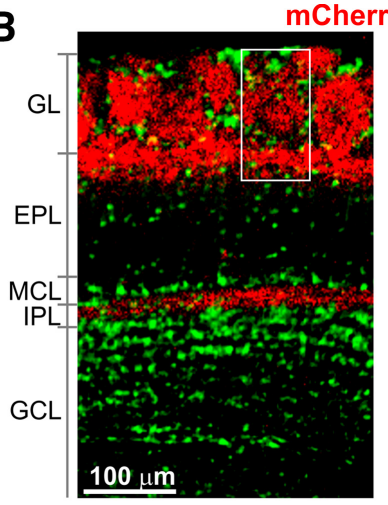

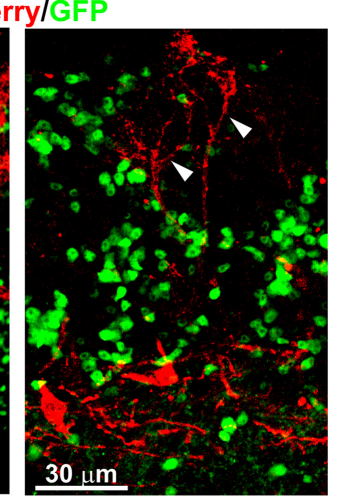
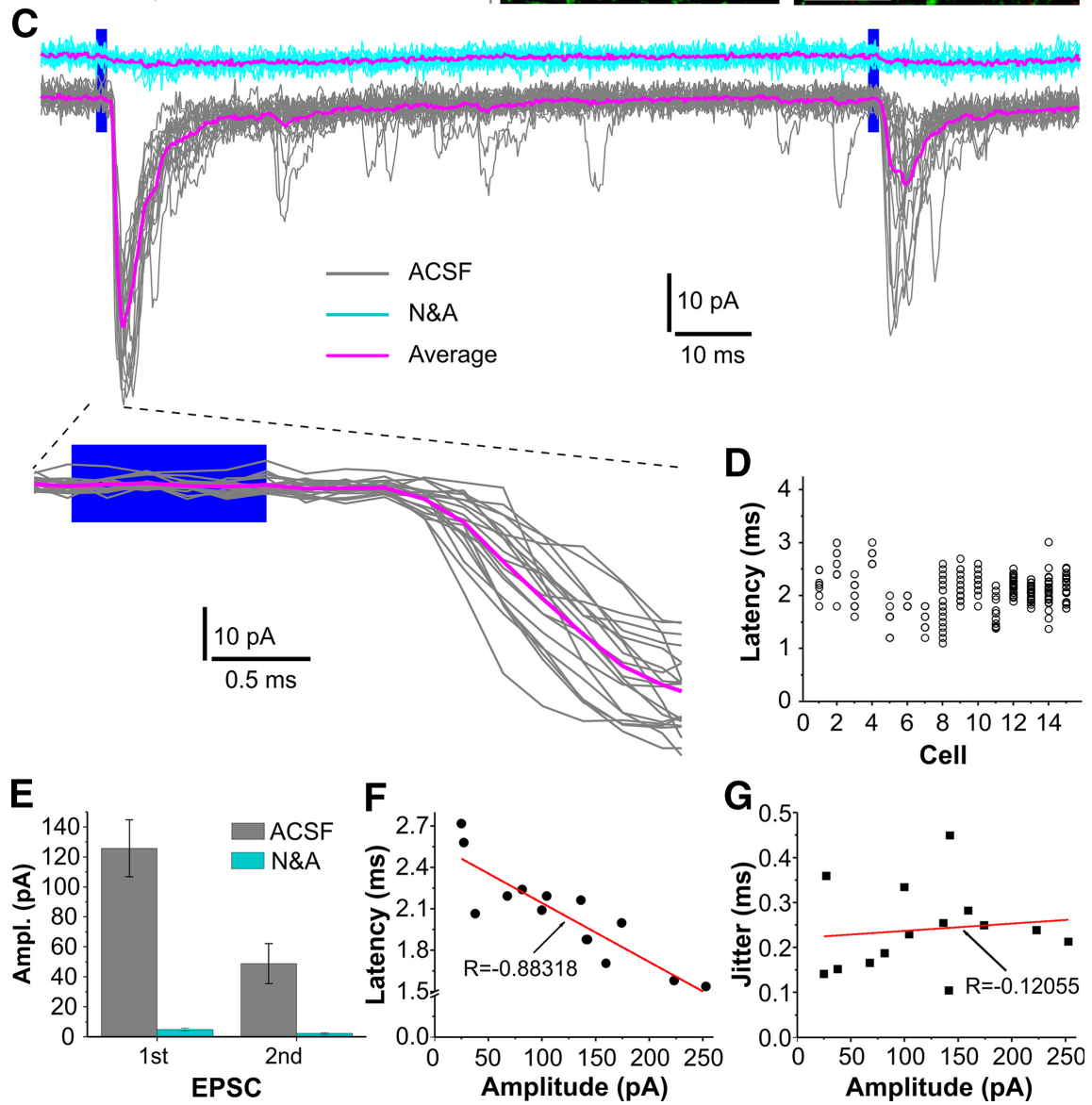

Figure 6. CCKergic TCs monosynaptically excite PGCs via dendrodendritic synapses. $\boldsymbol{A}$, Schematic showing experimental design. $\boldsymbol{B}$, left, Confocal images showing ChR2-mCherry expression in TCs and GAD65-GFP-tagged PGCs. Right, Blown-up from the left showing the relationship between the GFP-tagged PGCs and apical dendrites (white arrowheads) of mCherry-expressing TCs. C, top and middle, Typical voltage-clamp traces showing EPSCs recorded in a PGC in response to paired optical stimulation delivered to the PGC-affiliated glomerulus to activate CCKergic TC apical dendrites in ACSF (gray, middle) or in the presence of NBQX and APV (N\&A, top cyan traces). Bottom, Blown-up from the middle traces showing onset latencies of the first EPSCs. D, Symbol plot showing onset latencies of the TC-evoked EPSCs in 15 PGCS. $E$, Bar graph showing the average amplitude of the TC-evoked EPSCs in 15 PGCS in ACSF or in the presence of NBQX and APV (N\&A). $\boldsymbol{F}, \mathbf{G}$, Symbol plots showing that EPSC onset latency $(\boldsymbol{F})$ but not its SD (synaptic jitter, $\boldsymbol{G}$ ) has a negative linear correlation with the average EPSC amplitude in 14 PGCS (one outlier excluded).

Andresen, 2001). In contrast to latencies, amplitude of the IPLevoked EPSCs $(71.9 \pm 5.0 \mathrm{pA}, n=9)$ was larger than that of the superficial EPL-evoked ones $\left(43.0 \pm 2.9 \mathrm{pA}, n=9 ; t_{(8)}=\right.$ $10.25549, p=0.000007$, paired $t$ test; Fig. $4 E$ ). Despite their indistinguishable peak times $(1.8 \pm 0.4 \mathrm{~ms}$ for superficial EPL evoked vs $1.9 \pm 0.3 \mathrm{~ms}$ for IPL evoked, $n=9, t_{(8)}=0.7236, p=1$, paired $t$ test), the IPL-evoked EPSCs exhibited much smaller decay time constants $(31.2 \pm 2.7 \mathrm{~ms}, n=9)$ compared with the superficial EPL-evoked EPSCs $\left(50.0 \pm 3.4 \mathrm{~ms}, n=9 ; t_{(8)}=7.82771\right.$, $p=0.0005$, paired $t$ test; Fig. $4 D$, bottom right traces, $F$ ). In addition, superficial EPL-evoked EPSCs exhibited paired-pulse depression (PPD) with a paired-pulse ratio (PPR; EPSC2/ EPSC1) of $0.79 \pm 0.04(n=9)$ while IPL-elicited EPSCs showed paired-pulse facilitation (PPF; PPR $=1.3 \pm 0.07, n=9, t_{(8)}=$ $6.11663, p=0.0003$, paired $t$ test; Fig. $4 D$, top and middle gray traces, $G)$.

Taken together, these results support a glutamate-mediated monosynaptic transmission from CCKergic STCs to GCs. The difference in kinetics, strength, and short-term plasticity of the EPSCs evoked by stimulation of the superficial EPL and IPL resemble those for the distal or proximal excitatory synapses onto the same GCs (Balu et al., 2007), suggesting functional 
A

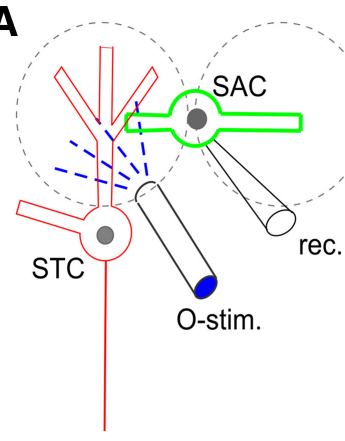

B

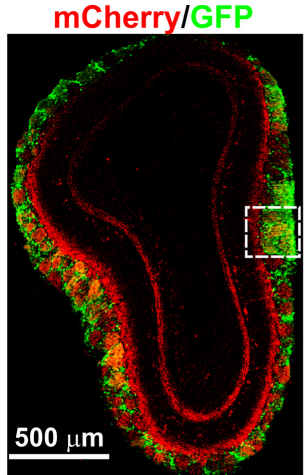

mCherry/GFP

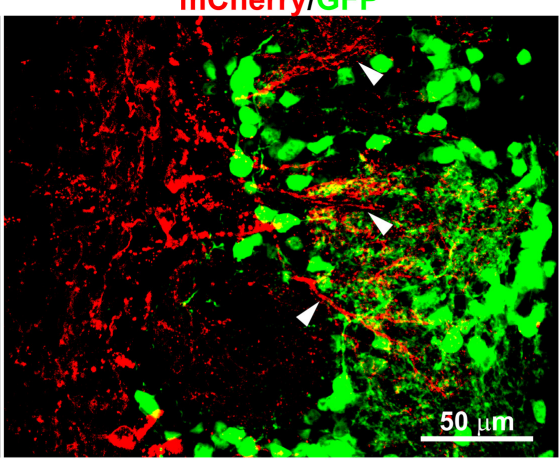

C
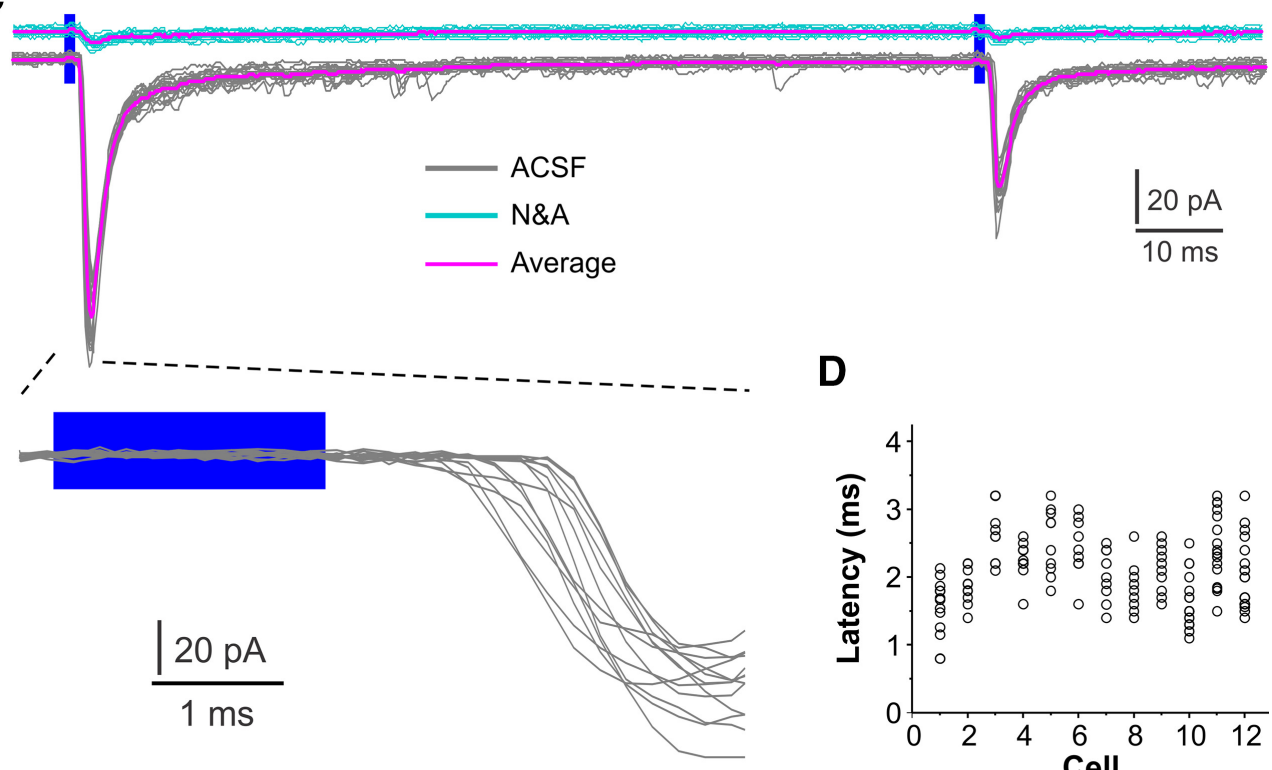

D

E

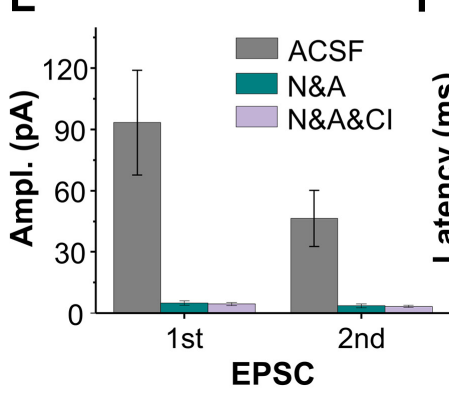

$\mathbf{F}$

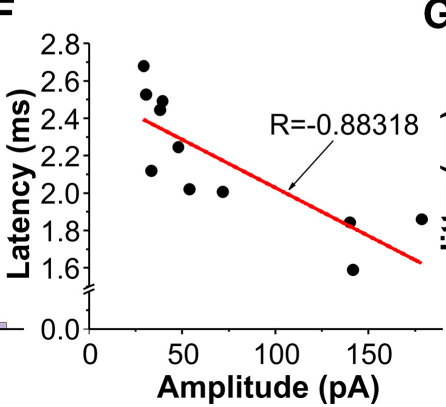

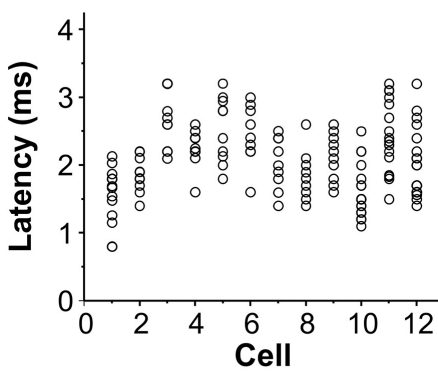

G

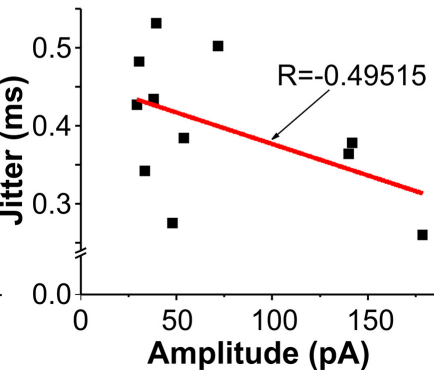

Figure 7. CCKergic TCS monosynaptically activate SACs via apical dendrites. $\boldsymbol{A}$, Schematic showing experimental design. $\boldsymbol{B}$, left, Confocal images of a coronal OB section showing ChR2mCherry expression in TCs and TH-GFP-tagged SACs. Right, Blown-up from the left showing the relationship of GFP-tagged SACS and mCherry-expressing TC apical dendrites (white arrowheads). C, top and middle, Typical voltage-clamp traces showing EPSCs recorded in a SAC in response to paired optical stimulation delivered to the SAC-affiliated glomerulus to activate CCKergic TC apical dendrites in ACSF (gray, middle) or in the presence of NBQX and APV (N\&A, top cyan traces). Bottom, Blown-up from the middle traces showing onset latencies of the first EPSCS. D, Symbol plot showing the TC apical dendrite-evoked EPSC onset latencies in 12 SACS. $E$, Bar graph showing the average amplitude of the TC-evoked EPSCs in 12 SACs in ACSF, with addition of NBQX and APV (N\&A), and further addition of CI988 (N\&A\&CI), a selective CCK-B receptor antagonist. $\boldsymbol{F}, \boldsymbol{G}$, Symbol plots showing that EPSC onset latency (F) but not its SD (synaptic jitter, $\boldsymbol{G}$ ) has a negative linear correlation with the average EPSC amplitude in 11 SACS (one outlier excluded).

dendrodendritic synapses formed between CCKergic TC lateral dendrites and GC distal apical dendrites in the superficial EPL, and axodendritic synapses established between CCKergic TC axon collaterals and GC proximal dendrites in the IPL.

The CCKergic IAS neurons terminate their axons in the IPL on the opposite side of the same bulb and form synaptic connections with local GCs (Liu and Shipley, 1994). To examine the function of these synapses, we injected AAV-ChR2-mCherry in the medial side of each bulb in CCK-Cre x GAD65-GFP mice with a volume to express ChR2 only in TCs on the medial side and sufficient level of $\mathrm{ChR} 2$ in the axons projected to the IPL on the lateral side of the same bulb (Fig. $5 A-C$ ). In these conditions, we selectively recorded from the GFP-expressing GCs on the lateral side and observed inward currents evoked by optical stimulation of IPL right above the recorded cells (Fig. $5 D$, bottom gray traces) but not of superficial EPL (Fig. $5 D$, top traces). These currents were abolished by NBQX and APV (Fig. $5 D$, middle blue traces, $G)$, supporting their EPSC nature. The average onset 

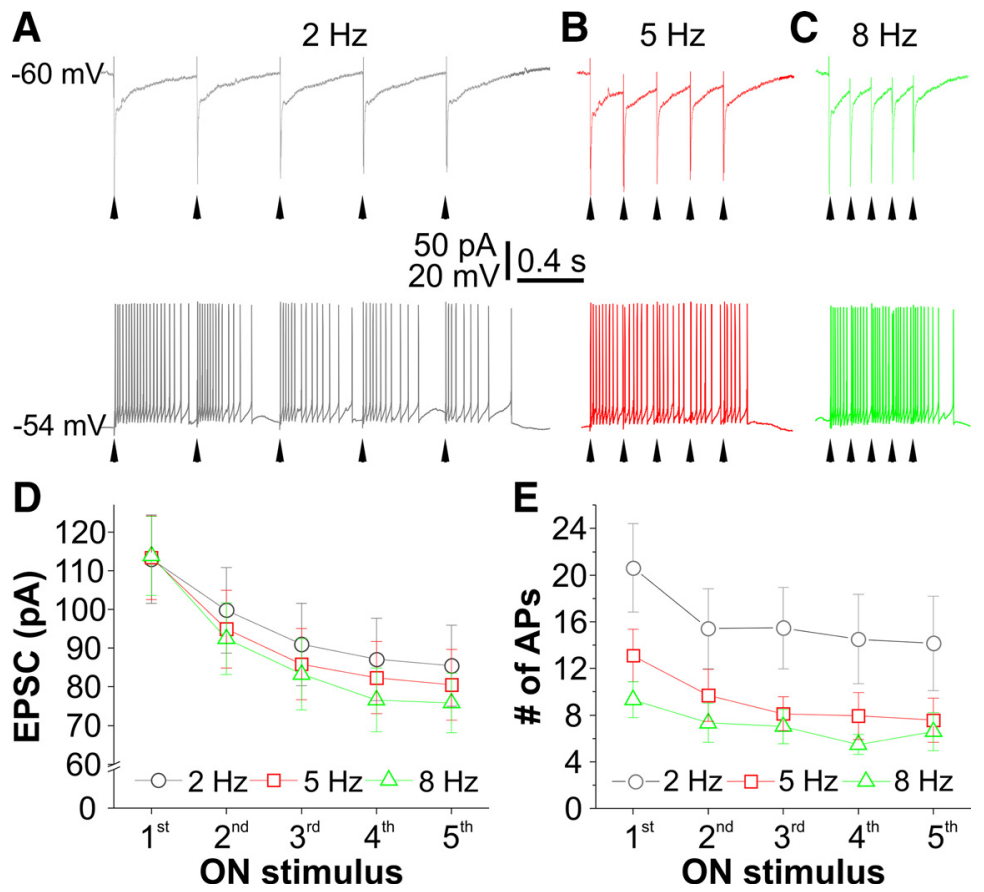

Figure 8. STC responses to sniff-like patterned $0 \mathrm{~N}$ train stimulation. $A-C$, top, Typical voltage-clamp traces showing EPSCs in a STC evoked by a train of five electrical stimuli delivered to the $0 \mathrm{~N}$ at $2 \mathrm{~Hz}(\boldsymbol{A}), 5 \mathrm{~Hz}(\boldsymbol{B})$, or $8 \mathrm{~Hz}(\boldsymbol{C})$. Bottom, Typical current clamp traces showing STC burst spike responses evoked by the same patterned train stimuli. $D, E$, Average data plot showing the amplitude of $\operatorname{EPSCS}(\boldsymbol{D} ; n=8$ cells) or number of action potentials (APs) per response $(\boldsymbol{E} ; n=6$ cells) evoked by trains of patterned $0 \mathrm{~N}$ stimuli at three frequencies.

latency of these EPSCs in nine cells was $2.0 \pm 0.13 \mathrm{~ms}(n=9)$ with a synaptic jitter of $373.8 \pm 29 \mu \mathrm{s}(n=9$; Fig. $5 E)$, indicating monosynaptic transmission from CCKergic TC axon to GCs in the IPL on the opposite side. The EPSC decay time constant $(\tau ; 9.3 \pm 0.1 \mathrm{~ms}, n=336$ traces in 9 cells; Fig. $5 F)$ was comparable to that of the IPL-evoked EPSCs in GCs on the same side. These EPSCs exhibited PPF (PPR $=1.7 \pm 0.1, n=9$; Fig. $5 D$, bottom gray traces, $G$ ). The consistent kinetics and short-term plasticity of EPSCs recorded in GCs on both the same and opposite sides of the CCKergic TCs suggest that CCKergic TCs form axodendritic synapses with GCs in the IPL on both sides of the same bulb.

In sum, our results revealed that CCKergic TCs released glutamate to drive GABAergic GCs via monosynaptic connections. These monosynaptic connections are established between the lateral dendrites of CCKergic TCs and GC distal apical dendrites in the superficial EPL, CCKergic TC axon collaterals or terminals in the IPL and GC proximal apical dendrites on both sides of each bulb. The dendrodendritic synapses and axodendritic synapses between CCKergic TCs and GCs exhibit differences in decay kinetics and short-term plasticity.

\section{CCKergic TCs drive inhibitory glomerular interneurons}

The ramification of STC apical dendrites in single glomeruli suggests potential dendrodendritic synapses between CCKergic STCs and the GABAergic PGCs or SACs, two major populations of local inhibitory interneurons with somas and dendrites or processes confined to the GL (Pinching and Powell, 1971; Aungst et al., 2003; Hayar et al., 2004b; Kiyokage et al., 2010). To test the possibility of synaptic transmission from CCKergic STCs to PGCs that exclusively express GAD65 (Kiyokage et al., 2010), a key enzyme for GABA biosynthesis, we applied the same optogenetic approach in CCK-Cre x GAD65-GFP mice for selective activation of the CCKergic TCs and recording from PGCs (Fig. 6A,B). As illustrated in Figure $6 A$, optical stimulation of the glomeruli affiliated by the recorded cells evoked inward currents in PGCs voltage clamped at $-60 \mathrm{mV}$ (Fig. $6 \mathrm{C}$, middle gray traces). These inward currents were abolished by bath-applied NBQX and APV (N\&A; Fig. $6 C$, top cyan traces, $6 E$ ), supporting that they are glutamate-mediated EPSCs. These EPSCs had short onset latencies ranging from 1.1 to $3.03 \mathrm{~ms}(2.2 \pm 0.1 \mathrm{~ms}, n=16$ cells $)$ and a synaptic jitter of $239.2 \pm 21.8 \mu$ s $(n=15$ cells; Fig. $6 C$, bottom blown-up trace, $6 D$ ). Based on the same criteria applied to GCs, these results corroborate a glutamate-mediated monosynaptic transmission from CCKergic TC apical dendrites to PGC dendrites. However, in contrast to the PPF of GC responses to paired optical stimuli of IPL, the EPSCs evoked by the identical pairedpulse stimuli of CCKergic TCs exhibited robust PPD in PGCs (Fig. 6C, middle traces) with a PPR of $0.42 \pm 0.1(n=15$; Fig. $6 E)$. Further analysis revealed a negative linear regression of the average onset latency $\left(F_{(1,12)}=42.54817\right.$, $p=2.83967 \times 10^{-5}$; Fig. $\left.6 F\right)$ but not the average latency SD (synaptic jitter; $F_{(1,12)}=0.17697$, $p=0.68142$; Fig. $6 G$ ) with amplitude of EPSCs.

The second population of inhibitory interneurons in the GL are SACs, which exclusively express the molecular marker, tyrosine hydroxylase (TH), a key enzyme for biosynthesis of dopamine. To examine potential synaptic transmission from CCKergic TCs to SACs, mCherry-ChR2 was expressed in OB CCKergic TCs of CCK-Cre $\mathrm{x}$ TH-GFP mice so that we were able to selectively identify SACs and record their responses to optical stimulation of CCKergic TCs in OB slices (Fig. 7A,B). Injection of AAV2.5-ChR2mCherry into the GL of each OB in CCK-Cre $x$ TH-GFP mice produced consistent expression of $\mathrm{ChR} 2$-mCherry predominantly in OB STCs (Fig. 7B). With this powerful approach, we found that optical stimulation of the glomeruli affiliated by the recorded cells elicited inward currents in all recorded SACs voltage clamped at $-70 \mathrm{mV}$ (Fig. $7 C$, middle gray traces). These inward currents were almost completely blocked by bath-applied NBQX and APV (Fig. 7C, top cyan traces, 7E), substantiating that they are CCKergic TC-evoked and glutamate-mediated EPSCs.

Since SACs are selectively activated by exogenously applied CCK via CCK-B receptors (Liu and Liu, 2018), we tested whether the residual NBQX/APV-resistant inward currents were mediated by activation of CCK-B receptors. However, addition of CI988 $(10 \mu \mathrm{M})$, a selective CCK-B receptor antagonist (Hughes et al., 1990), did not change the residual small inward currents (Fig. $7 E$ ), indicating that they are not due to endogenous CCK activation of CCK-B receptors.

Like those recorded in PGCs, the CCKergic TC-evoked EPSCs in SACs had short onset latencies ranging from 1.0 to $3.8 \mathrm{~ms}(2.1 \pm 0.2 \mathrm{~ms}, n=12$ cells $)$ and a synaptic jitter of $445.3 \pm 48.9 \mu \mathrm{s}(n=120$ traces from 12 cells; Fig. $7 C$, bottom blown-up traces, $7 D$ ), suggesting monosynaptic transmission from CCKergic TCs to SACs. Additionally, the CCKergic TCevoked EPSCs in SAC exhibited PPD (Fig. $7 C$, middle gray traces) with a PPR of $0.58 \pm 0.1(n=12$; Fig. $7 E)$. Further analysis revealed a negative correlation of the average EPSC onset latency $\left(F_{(1,9)}=17.50628, p=0.00236\right.$, ANOVA test; Fig. $\left.7 F\right)$ but not the 
synaptic jitter $\left(F_{(1,9)}=2.92333, p=0.12148\right.$; Fig. $7 G$ ) with amplitude of EPSCs in 11 cells (one outlier excluded).

Collectively, our results demonstrate that both PGCs and SACs receive monosynaptic input from CCKergic TC apical dendrites. This direct synaptic input results in postsynaptic excitatory responses which exhibit the short-term plasticity PPD in both interneuron types.

\section{STC responses to repetitive on stimulation}

Sniffing actively brings odorants to repetitively activate OSNs (Welker, 1964; Verhagen et al., 2007; Wachowiak, 2011). Rodents increase sniff frequency during active investigation of novel odor stimuli (Welker, 1964; Macrides, 1975; Freeman et al., 1983; Youngentob et al., 1987; Verhagen et al., 2007). The functional significance of this behavioral alteration to olfaction is not fully understood. One possibility is to shape signal processing in downstream brain centers including the $\mathrm{OB}$ (Verhagen et al., 2007; Carey and Wachowiak, 2011). To test how CCKergic STCs respond to sniff-like patterned activation of OSNs, STCs in OB slices were voltage clamped at $-60 \mathrm{mV}$, while the $\mathrm{ON}$ received a train of five electrical stimuli every $15 \mathrm{~s}$ at three different intratrain frequencies, 2,5 , or $8 \mathrm{~Hz}$, which resemble resting breathing rate and low or high frequencies of exploratory sniffing, respectively. Consistent with the high glutamate release probability of ON terminals (Murphy et al., 2004; Vaaga and Westbrook, 2017), a train of five ON stimuli at any of these three frequencies elicited five progressively decreased EPSCs in all recorded STCs (Fig. 8A-C, top traces, $D$ ). For $2-\mathrm{Hz}$ stimulation, the average amplitude of the first, third, and fifth EPSC in eight cells was $113.0 \pm 11.4 \mathrm{pA}, 91.0 \pm 10.6 \mathrm{pA}\left(t_{(28)}=\right.$ $12.60077, p=4.66487 \times 10^{-12}$ compared with the first, ANOVAOneWayRM with Bonferroni comparison), and $85.4 \pm 10.5 \mathrm{pA}\left(_{(28)}=15.75812\right.$, $p=.89033 \times 10^{-14}$ compared with the first; $t_{(28)}=3.15735, p=0.03792$ compared with third, ANOVAOne WayRM with Bonferroni comparison). Interestingly, this synaptic depression did not show significant frequency dependence as in ETCs and MCs (Vassar et al., 1994), indicating that STCs convey OSN signals to postsynaptic targets with higher fidelity (Short and Wachowiak, 2019).

To assess the repetitive $\mathrm{ON}$ stimulation-evoked STC spike response, a more physiologically relevant measure of potential impact on postsynaptic targets, we repeated the above experiment in current clamp in a separate set of STCs. As shown in Figure $8 A-C$, bottom traces, STCs responded to repetitive ON stimulation with bursts of action potentials. Because of the long duration of each spike burst, responses were only distinguishable for stimuli at $2 \mathrm{~Hz}$ but not for 5 or $8 \mathrm{~Hz}$ (Fig. $8 A-C$, bottom traces). Since the interstimulus interval of the $5-$ and $8-\mathrm{Hz}$ train stimuli was shorter than the duration of individual spike burst response in STCs, the repetitive spike responses merged together and appeared as a continuous spiking pattern (Fig. $8 B, C$, bottom traces) at these two stimulation frequencies. Consequently, the number of action potentials in each burst response decreased as
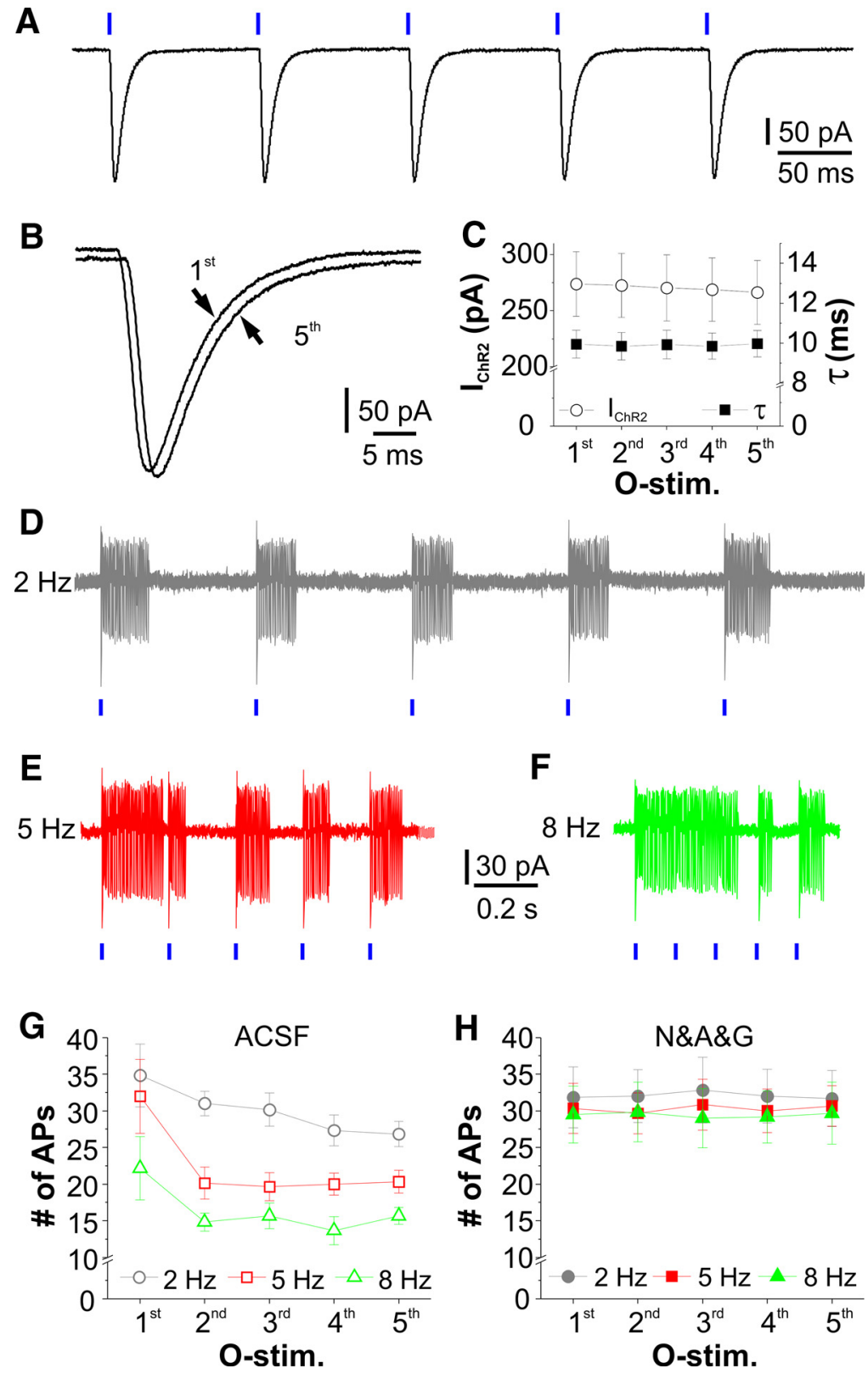

Figure 9. Rhythmic activation of ChR2 produces high-fidelity excitatory responses in CCKergic STCs. A, Typical voltage-clamp trace showing ChR2 current in a CCKergic STC evoked by five optical stimuli at $10 \mathrm{~Hz}$ in the presence of TTX to block action potential-dependent circuit influence. $B$, The first and the fifth ChR2 current $\left(\mathrm{I}_{\mathrm{ChR2}}\right)$ traces superimposed to show the kinetic fidelity. C, Symbol plot showing consistency of the amplitude and decay time constant $(\tau)$ of ChR2 currents evoked by a train of five optical stimuli at $10 \mathrm{~Hz}$ in eight cells. $\boldsymbol{D}-\boldsymbol{F}$, Typical cellattached recording traces showing spike responses of a CCKerigc STC to the patterned train stimulation at $2 \mathrm{~Hz}$ $(\boldsymbol{D}), 5 \mathrm{~Hz}(\boldsymbol{E})$, or $8 \mathrm{~Hz}(\boldsymbol{F}) . \mathbf{G}, \boldsymbol{H}$, Average data plots showing the number of action potentials evoked by each of the five optical stimuli at three different frequencies in six STCS in ACSF (G) or in the presence of NBQX, APV, and gabazine (D\&A\&G, $\boldsymbol{H})$.

stimulation frequencies increased from 2 to $8 \mathrm{~Hz}$ ( $n=6$ cells; Fig. $8 E$ ). Consistent with voltage-clamp responses, the number of action potentials evoked by each train stimulation progressively decreases from the first to the fifth across three frequencies (Fig. 8, compared $E$ with, $D$ ), suggesting that sniff-like patterned stimulation of STCs triggers progressively depressed responses in their postsynaptic targets.

Repetitive optogenetic excitation of CCKergic STCs

Given the high transmitter release probability of ON terminals (Murphy et al., 2004; Vaaga and Westbrook, 2017), the adaptation 


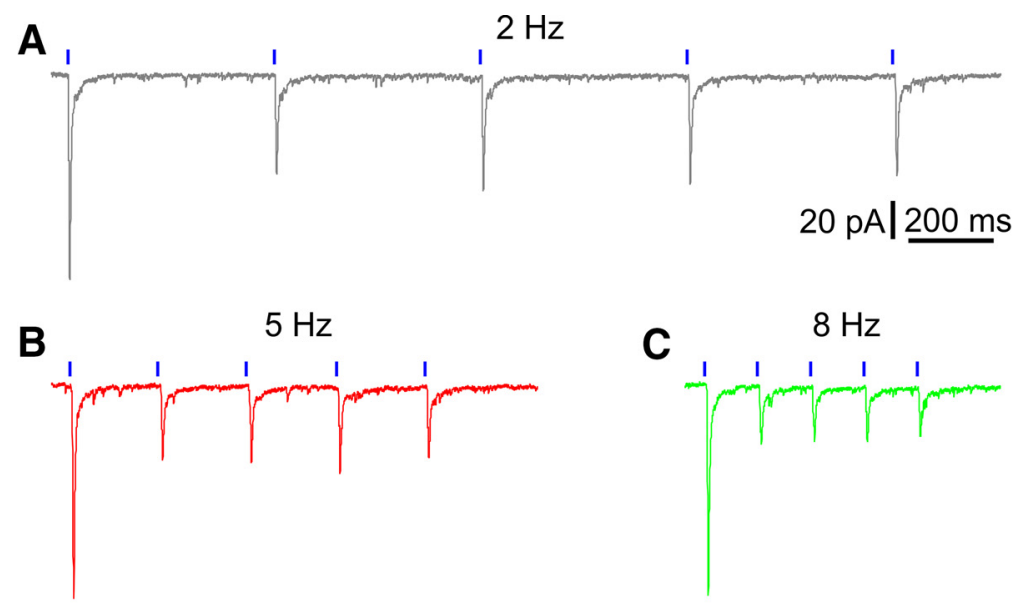

to examine CCKergic STC intrinsic responses to repetitive stimulation.

We first assessed the fidelity of repetitive optical stimulation-evoked ChR2 currents in STCs in OB slices in the presence of TTX to eliminate the action potential-dependent circuit influence. Under these conditions, ChR2 currents were recorded from STCs in voltage clamp $\left(\mathrm{V}_{\mathrm{h}}=\right.$ $-60 \mathrm{mV}$ ) in response to five brief (2-ms) repetitive optical stimuli at $10 \mathrm{~Hz}$ (Fig. 9A). Neither amplitude nor kinetic profile of the repetitively evoked ChR2 currents varied from one to another $(n=8$ cells; Fig. $9 A-C)$. These findings demonstrated high fidelity of ChR2 currents in CCKergic STCs in response to repetitive stimulation in the absence of circuit influence. Thus,
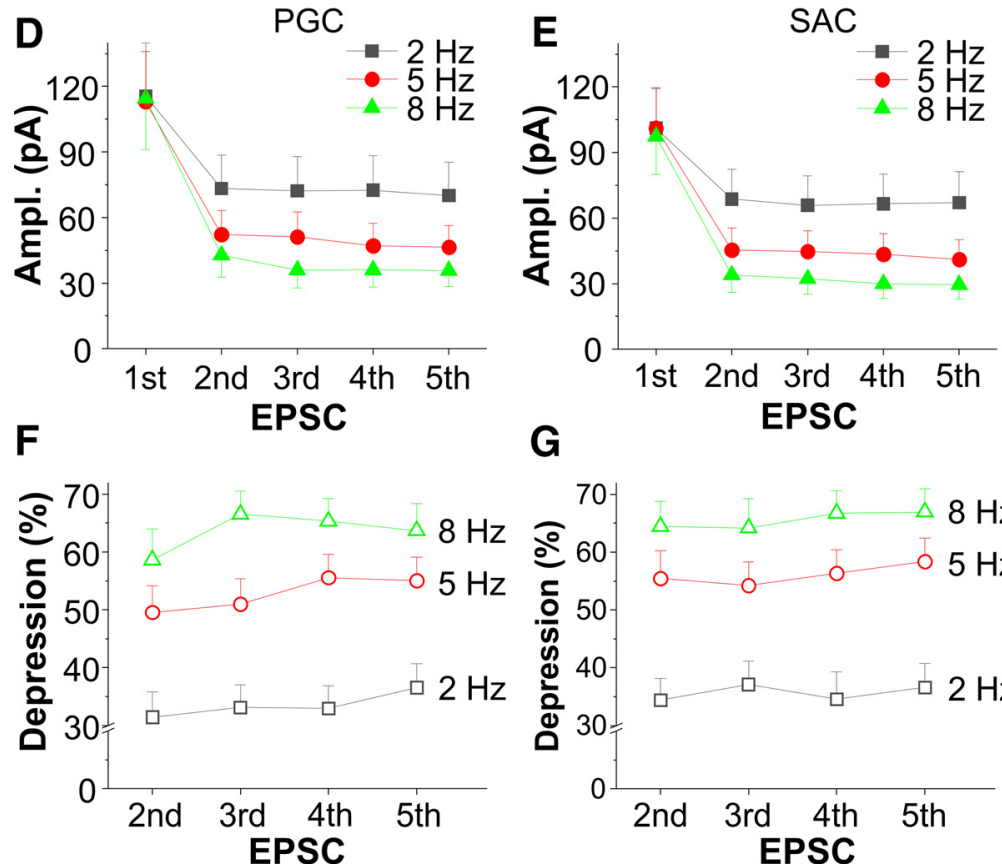
this optogenetic approach enabled us to test STC intrinsic spike responses to repetitive stimulation. To this end, repetitive optic stimulation-evoked spike responses were recorded in the ChR2-EYFP-expressing STCs in OB slices in the cell-attached mode, which preserves the intracellular environment and intrinsic properties of the recorded cells. As shown in Figure 9D-F, a train of five laser light pulses (2-ms duration) at 2,5 , or $8 \mathrm{~Hz}$ elicited bursts of spikes in STCs in ACSF. Similar to the repetitive ON stimulation-evoked STC responses (Fig. 8), the number of spikes ( $n=6$ cells; Fig. $9 G$ ) per response exhibited a trending adaptation, i.e., the number of spikes elicited by the first optical stimulus was higher than those by the subsequent stimuli. For $2-\mathrm{Hz}$ stimulation, the average spike number of the first, second, and fifth response in six cells was $34.8 \pm 4.3,31.0 \pm 1.7\left(t_{(20)}=1.12287, p=1\right.$ compared with first, ANOVAOneWayRM with Bonferroni comparison), and $26.8 \pm 1.7\left(t_{(20)}=\right.$ 2.34339, $p=0.29556$ compared with first; $t_{(20)}=$ 1.22051, $p=1$ compared with second; ANOVA OneWayRM with Bonferroni comparison), respectively. For $5-\mathrm{Hz}$ stimulation, the average spike number of the first, second, and fifth response in six cells was $32.0 \pm 5.1,20.2 \pm 2.2$ $\left(t_{(20)}=3.69391, p=0.01437\right.$ compared with first, ANOVAOneWayRM with Bonferroni comparison), and $20.3 \pm 1.6\left(t_{(20)}=3.64188, p=\right.$

of CCKergic STC responses to repetitive ON stimulation can be interpreted as a reflection of gradual decrease in presynaptic neurotransmitter release. Alternatively, STC intrinsic properties and/or circuit influence may also contribute to this since ON stimulation can directly and indirectly engage inhibitory glomerular interneurons to release GABA or dopamine (Wellis and Scott, 1990; Aungst et al., 2003; Murphy et al., 2004; Maher and Westbrook, 2008; Gire and Schoppa, 2009; Shao et al., 2009; Kiyokage et al., 2010; Borisovska et al., 2013; Liu et al., 2013; Najac et al., 2015), which activates $G_{A B A}$ receptors or dopamine D2 receptors on ON terminals and inhibits glutamate release (Nickell et al., 1994; Wachowiak and Cohen, 1999; Aroniadou-Anderjaska et al., 2000; Ennis et al., 2001; Pírez and Wachowiak, 2008; McGann, 2013). To test these possibilities, we characterized CCKergic STC responses to sniff-like patterned optogenetic activation. This approach combined with pharmacological tools to minimize circuit influence allowed us
0.01622 compared with first; $t_{(20)}=0.05203, p=1$ compared with second; ANOVAOneWayRM with Bonferroni comparison), respectively.

Given that GABAergic GCs, PGCs, and SACs receive direct synaptic input from CCKergic STCs, another possibility is that inhibitory feedback from these inhibitory interneurons via potential reciprocal synapses weakens the second to the fifth spike responses in STCs. To test this, we repeated the above experiments in the presence of the fast synaptic transmission blockers NBQX, APV and GBZ to block the potential reciprocal synaptic interactions between STCs and GABAergic inhibitory interneurons. In these conditions, five repetitive optical stimuli at 2,5 , or $8 \mathrm{~Hz}$ elicited essentially identical number of action potentials in all recorded ChR2-expressing STCs $(n=6$ cells; Fig. $9 H$ ), suggesting that inhibitory feedback from local inhibitory interneurons rather than intrinsic 
properties contributes to the repetitive stimulation-evoked STC spike response depression.

Taken together, our results demonstrate that repetitive optogenetic activation resembles sniff-like patterned ON stimulation in triggering CCKergic STC spike responses, which exhibit a trending adaptation at least partially due to inhibitory synaptic feedback from local GABAergic interneurons.

\section{Repetitive STC activation produces dynamically different synaptic responses in GCs and inhibitory glomerular interneurons}

The axodendritic synapses between TC axon collaterals and proximal dendrites of GCs in the IPL exhibited PPF, while the dendrodendritic synapses between TC lateral dendrites and distal dendrites of GCs in the superficial EPL or between STC apical dendrites and PGCs or SACs in the GL showed PPD, indicating that the CCKergic STC-driven inhibitory strength shifts from superficial to deep levels in the $\mathrm{OB}$ in response to repetitive $\mathrm{ON}$ input. To test this, we recorded from PGs, SACs, or GCs in voltage clamp in response to repetitive activation of CCKergic STCs by a train of five optic stimuli at 2,5 , or $8 \mathrm{~Hz}$ as described above. In these conditions, both PGs and SACs similarly responded to the train stimulation across all three different frequencies with synaptic depression. Specifically, the second to the fifth EPSCs were dramatically smaller than the first EPSC across all three frequencies (Fig. 10A-E). The depression level depended on stimulation frequencies. The second EPSC evoked by the 2-, $5-$, and $8-\mathrm{Hz}$ train stimulation in 19 PGCs was depressed by $31.5 \pm 4.4 \%, 49.6 \pm 4.6 \%\left(t_{(36)}=5.06715, p=3.67042 \times 10^{-5}\right.$ compared with $2 \mathrm{~Hz}$, ANOVAOneWayRM with Bonferroni comparison), and 58.6 $\pm 5.3 \%\left(t_{(36)}=7.60105, p=1.62927 \times\right.$ $10^{-8}$ compared with $2 \mathrm{~Hz} ; t_{(36)}=2.5339, p=0.04732$ compared with $5 \mathrm{~Hz}$, ANOVAOneWayRM with Bonferroni comparison), respectively, whereas the fifth EPSC in the same groups of cells was depressed by $36.6 \pm 4.1 \%, 55.0 \pm 4.0 \%\left(_{(36)}=4.8395\right.$, $p=7.35327 \times 10^{-5}$ compared with $2 \mathrm{~Hz}$, ANOVAOneWayRM with Bonferroni comparison), and $63.7 \pm 4.7 \%\left(t_{(36)}=7.10365\right.$, $p=7.20448 \times 10^{-8}$ compared with $2 \mathrm{~Hz} ; t_{(36)}=2.26415, p=$ 0.08906 compared with $5 \mathrm{~Hz}$, ANOVAOneWayRM with Bonferroni comparison), respectively (Fig. 10F).

Similarly, the second EPSC evoked by the 2-, 5-, and 8- Hz train stimulation in 15 SACs was depressed by $34.4 \pm 3.8 \%, 55.4 \pm$ $4.8 \%\left(t_{(28)}=6.11809, p=3.9981 \times 10^{-6}\right.$ compared with $2 \mathrm{~Hz}$, ANOVAOneWayRM with Bonferroni comparison), and $64.5 \pm$ $4.3 \%\left(t_{(28)}=8.75217, p=5.02164 \times 10^{-9}\right.$ compared with $2 \mathrm{~Hz}$; $t_{(28)}=2.63408, p=0.04076$ compared with $5 \mathrm{~Hz}$, ANOVAOne WayRM with Bonferroni comparison), respectively. The fifth EPSC in the same groups of cells was depressed by $36.6 \pm 4.1 \%$, $58.4 \pm 4.1 \%\left(t_{(28)}=7.86742, p=4.30664 \times 10^{-8}\right.$ compared with $2 \mathrm{~Hz}$, ANOVAOneWayRM with Bonferroni comparison), and $66.9 \pm 4.0 \%\left(t_{(28)}=10.96717, p=3.62115 \times 10^{-11}\right.$ compared with $2 \mathrm{~Hz} ; t_{(28)}=3.09905, p=0.01315$ compared with $5 \mathrm{~Hz}$, ANOVAOne WayRM with Bonferroni comparison), respectively (Fig. 10G). But there was no significant difference from the second to the fifth EPSCs within each frequency.
For GCs, we tested only the axodendritic synapses formed between CCKergic TC axons and GC on the opposite side of the same bulb as illustrated by Figure 11A. In contrast to PGCs and SACs, GCs responded with facilitated EPSCs across all three frequencies (Fig. 11B-D). Interestingly, the third EPSC reached the maximal amplitude then maintained at a steady level across three train stimulation frequencies (Fig. 11E). The facilitation level depends on intratrain stimulation frequencies. The amplitude of the second EPSC evoked by the 2-, 5-, or $8-\mathrm{Hz}$ train stimulation in 15 GCs increased by $13.5 \pm 5.1 \%, 39.3 \pm 8.3 \%\left(t_{(28)}=2.72161\right.$, $p=0.03314$ compared with $2 \mathrm{~Hz}$, ANOVAOneWayRM with Bonferroni comparison), or $48.2 \pm 7.6 \%\left(t_{(28)}=3.6534, p=\right.$ 0.00317 compared with $2 \mathrm{~Hz} ; t_{(28)}=0.93179, p=1$ compared with $5 \mathrm{~Hz}$, ANOVAOneWayRM with Bonferroni comparison) compared with the first EPSC, respectively (Fig. 11E); meanwhile, the amplitude of the fifth EPSC evoked by the same train stimulations increased by $13.6 \pm 5.3 \%, 46.3 \pm 10.3 \%\left(t_{(28)}=\right.$ 2.82003, $p=0.02617$ compared with $2 \mathrm{~Hz}$, ANOVAOneWayRM with Bonferroni comparison), or $59.5 \pm 10.0 \%\left(t_{(28)}=3.96492\right.$, $p=0.00138$ compared with $2 \mathrm{~Hz} ; t_{(28)}=1.14489, p=0.78584$ compared with $5 \mathrm{~Hz}$, ANOVAOneWayRM with Bonferroni comparison) for 2, 5, or $8 \mathrm{~Hz}$, respectively (Fig. 11F).

In sum, our results showed that repetitive activation of the CCKergic TCs engaged both populations of inhibitory glomerular interneurons via their apical dendrites and GCs via their axons in the IPL on the opposite side of the same bulb but the inhibitory strength progresses in opposite directions at these two distinct locations during repetitive stimulation, i.e., waning in the glomerular circuits but waxing in the IPL.

\section{Discussion}

We characterized the dynamic functions of synaptic pathways from CCKergic TCs to three distinct populations of inhibitory interneurons in the $\mathrm{OB}$ with the optogenetic approach leading to four major findings (Fig. 12). First, the vast majority of CCKexpressing neurons in the $\mathrm{OB}$ are STCs, which receive direct 


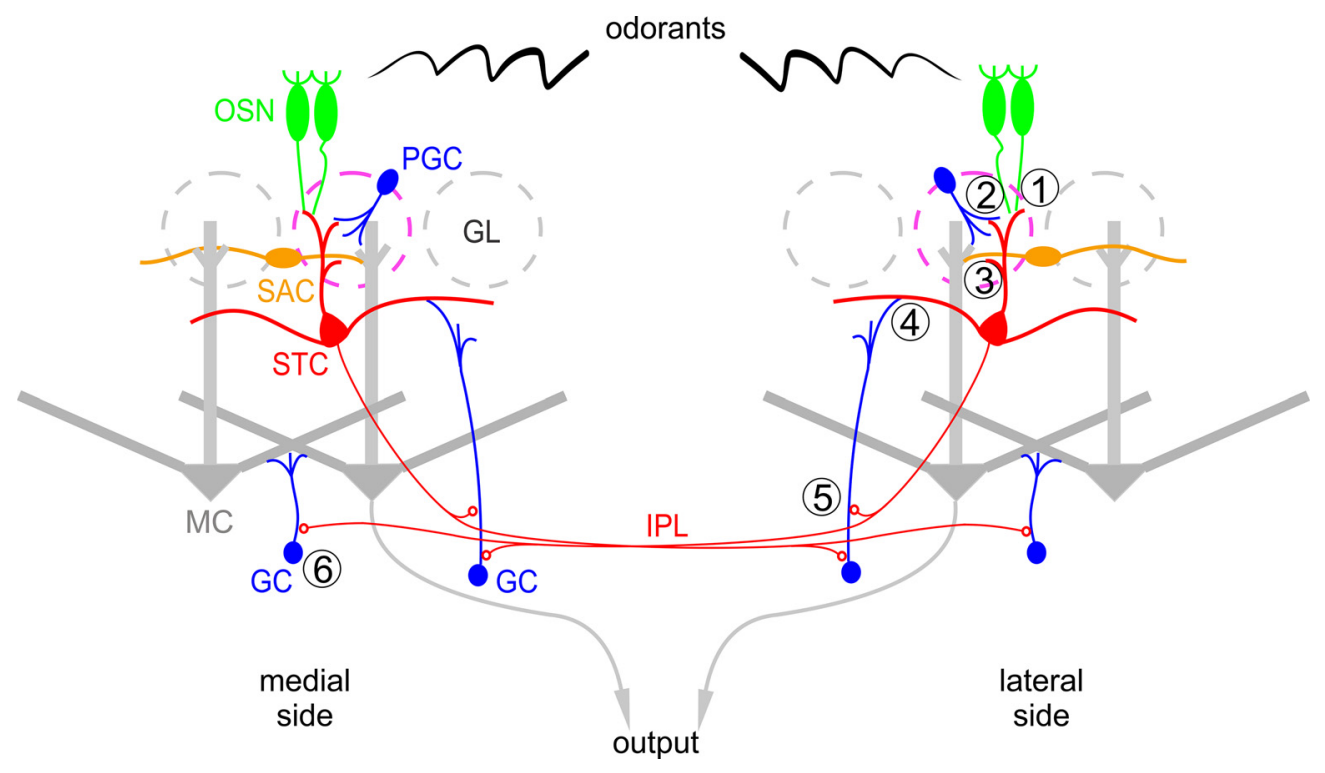

Figure 12. Summary of CCKergic TC actions on two spatially segregated inhibitory circuits in the OB. STCs receive direct excitatory input from OSNs (1) and intermediate the excitatory input to the GABAergic PGCS (2) and the GABAergic/dopaminergic SACS (3) via dendrodendritic synapses onto their apical dendrites in the GL. One the other hand, STCs activate the distal dendrites of GCs via dendrodendritic synapses onto their lateral dendrites in the superficial EPL (4) and excite proximal dendrites of GC on the same (5) and opposite sides (6) of the same bulb via axodendritic synapses onto their axon collaterals and terminals in the IPL, respectively. In response to sniff-like patterned stimulation, the dendrodendritic synapses exhibit frequency-dependent depression, while the axodendritic synapses are subject to frequency-dependent facilitation.

synaptic input from OSNs. This glutamate-mediated monosynaptic transmission exhibits slight depression in response to repetitive sniff-like patterned stimulation of OSNs. Second, CCKergic TCs form two functionally distinct types of monosynaptic connections with GCs: (1) dendrodendritic synapses onto GC distal dendrites via lateral dendrites in the superficial EPL; (2) axodendritic synapses onto GC proximal dendrites via axons collaterals or terminals in the IPL on both sides of each bulb. The dendrodendritic synapses show slow functional kinetics and PPD, while the axodendritic transmission exhibits fast kinetics and PPF. Third, CCKergic TCs monosynaptically excite both PGCs and SACs, two major populations of inhibitory glomerular interneurons. These glutamatergic synapses exhibit robust PPD. Finally, repetitive activation of CCKergic TCs frequency dependently shifts $\mathrm{OB}$ inhibitory strength from the superficial glomerular to deep IPL levels.

\section{Optogenetic labeling CCKergic TCs in the OB}

Our predominant expression of ChR2-EYFP in the CCKergic STCs is consistent with previous immunostaining findings that somata of CCK immunoreactive neurons mainly reside in the superficial EPL but sparsely in the deep EPL (Seroogy et al., 1985; Liu and Shipley, 1994). However, slightly different patterns of CCKergic TC distribution were observed in other studies, in which relatively more deep TCs (DTCs) were labeled by Cre-dependent expression of fluorescent proteins (FPs) in the same transgenic CCK-Cre mouse line as we used (Marks et al., 2006; Cheetham et al., 2015; Economo et al., 2016; Short and Wachowiak, 2019). While the exact reasons for this discrepancy are unknown, there are a few of potential contributing variables. (1) Cre gene copies: our experiments were performed in CCKCre heterozygotes, while the same line but homozygotes were used in other studies (Marks et al., 2006; Cheetham et al., 2015; Economo et al., 2016; Short and Wachowiak, 2019). It is plausible that DTCs in CCK-Cre heterozygotes contain too low level of Cre to express detectable FPs. (2) FP expression approaches: we employed viral transfection, while others cross-bred CCK-Cre homozygotes with the Ai95 GCaMP6f reporter line (Economo et al., 2016; Short and Wachowiak, 2019). In that context, neurons expressing transient levels of CCK mRNA during embryonic or postnatal developing stages are potentially labeled. (3) Animal age: we injected virus in adult mice, while others injected virus in mice at their postnatal days 0-3 (Marks et al., 2006; Cheetham et al., 2015) when developing neurons with transient expression of CCK could be labeled. (4) Viral serotypes: we used AAV2.5, while studies of Marks et al. (2006) and Cheetham et al. (2015) employed AAV2.1, which transfects neurons with higher level of FP expression than AAV2.5 and shows anterograde transsynaptic tagging (Watakabe et al., 2015; Zingg et al., 2017).

\section{CCKergic TCs drive inhibitory glomerular interneurons}

Previous electrophysiological studies demonstrate that majority of PGCs are driven by ETCs (ETC-driven; De Saint Jan et al., 2009; Gire and Schoppa, 2009; Shao et al., 2009; Najac et al., 2015). The present study presented evidence that GAD65expressing PGCs received monosynaptic input from the glutamatergic CCKergic TCs other than ETCs. This is consistent with a recent study showing that a small population of PGCs labeled by GFP expression under the control of the Kv3.1 potassium channel promoter are monosynaptic excited by TCs, suggesting that PGCs are driven not only by ETCs but also by other TCs (Najac et al., 2015). More importantly, our study provided new evidence that the CCKergic TC-PGC synapse exhibited robust frequency-dependent depression in response to repetitive snifflike patterned stimulation, indicating that sniff-driven sensory input dynamically modulates glomerular inhibition from PGCs.

Like PGCs, vast majority of SACs are ETC-driven (Hayar et al., 2004b; Kiyokage et al., 2010). Our study provides the first evidence showing that the CCKergic TCs also monosynaptically drive SACs, adding another level of complexity to the SACformed circuitry in the OB. Furthermore, the CCKergic TC-SAC transmission is subject to robust short-term depression in 
response to paired-pulse or a train of repetitive presynaptic stimulation across frequencies resembling active sniffing (Welker, 1964; Youngentob et al., 1987; Verhagen et al., 2007). Despite the slight difference in depression level, the similar temporal profile across stimuli and frequency dependence of synaptic depression between the TC-PGC and TC-SAC pathways imply high probability of neurotransmitter release from these TC apical dendritic terminals.

Our previous study demonstrates that exogenous CCK selectively activates SACs (Liu and Liu, 2018), indicating that activation of CCKergic TCs produces CCK receptor-mediated excitatory postsynaptic responses in SACs. However, this was not observed in the present study. One interpretation is that CCK like other neuropeptides is released from STC apical dendritic terminals by an extremely small quantity (van den Pol, 2012) to produce a small and slow depolarization in SAC dendritic terminals that might not be detectable at SAC somata. Future work may be needed to directly measure CCK release in response to optogenetic activation of CCKergic TCs with the sensitive enzyme immunoassay (Dao et al., 2019).

\section{Dendrodendritic and axodendritic transmission from TCs to GCs}

We made two major findings in characterizing synaptic transmission from CCKergic STCs to GCs. First, CCKergic TCs drive GCs on the opposite side of the same bulb via axodendritic synapses in the IPL. This is consistent with previous anatomic and ultrastructural studies (Price and Powell, 1970b; Orona et al., 1984; Liu and Shipley, 1994), in which it was unclear whether the observed synaptic connections were established between TC axon collaterals and GCs in the IPL on the same side or on the opposite side of the bulb. The fast functional kinetics and short-term facilitation of this glutamatergic monosynaptic transmission from CCKergic STCs to GCs are in line with those of the excitatory axodendritic synapses onto GC proximal dendrites (Balu et al., 2007). Our GC reconstruction further supports this conclusion. Second, CCKergic STCs formed both dendrodendritic and axodendritic synapses respectively in the superficial EPL and IPL with the same population of superficial GCs on the same side of the bulb. These findings corroborate previous anatomic or ultrastructural studies (Price and Powell, 1970a,b; Jackowski et al., 1978; Orona et al., 1983; Liu and Shipley, 1994). Although the spatially segregated positioning of optical stimulation cannot absolutely dissociate GC responses mediated by the dendrodendritic synapses in the superficial EPL from those by axodendritic synapses in the IPL due to potential orthodromic and antidromic propagation of excitation between STC lateral dendrites and axons as revealed in MCs (Charpak et al., 2001; Margrie et al., 2001), at least three pieces of evidence support these responses were mediated by two distinct types of synapses in the same GCs: (1) the fast kinetics and short-term facilitation of the IPL-evoked responses in GCs on the same side is consistent with those of the IPL-evoked responses in GCs on the opposite side that is no doubt mediated by axodendritic synapses due to the lack of lateral dendritic activation; (2) the different kinetics and short-term plasticity of the superficial EPL- and IPL-evoked GC responses correspondingly resemble those of the excitatory dendrodendritic and axodendritic synapses onto the distal and proximal dendrites of GCs revealed by two-photon guided minimal stimulation (Balu et al., 2007); (3) consistently, our neuron reconstruction reveals the recorded GCs as the superficial type, which have proximal dendrites crossing the IPL and distal dendrites reaching the superficial EPL (Orona et al., 1983).

\section{Functional implications}

The robust frequency-dependent depression at the synapses between CCKergic TCs and inhibitory glomerular interneurons suggests that CCKergic TCs function to relieve (disinhibit) intraglomerular and lateral inhibition of MCs thus increasing the system's sensitivity when animals raise sniffing frequency to investigate novel odors as PGCs and SACs powerfully suppress $\mathrm{MC}$ output through intraglomerular and interglomerular inhibition, respectively (Murphy et al., 2005; Gire and Schoppa, 2009; Shao et al., 2012; Banerjee et al., 2015; Najac et al., 2015; Liu et al., 2016; Short and Wachowiak, 2019). Furthermore, CCKergic TCs may regulate theta oscillations in the $\mathrm{OB}$ by driving inhibitory glomerular neurons, key contributors to this sniff-entrained population level of synchronized interactive activities between excitatory and inhibitory neurons (Macrides and Chorover, 1972; Margrie and Schaefer, 2003; Wachowiak, 2011; Fukunaga et al., 2014).

Via dendrodendritic and axodendritic synapses with GCs, CCKergic TCs could impact lateral inhibition of MCs, which is generally attributed to the reciprocal synapses between MC lateral dendrites and GC apical dendrites in the EPL (Rall et al., 1966; Burton, 2017). Additionally, the frequency-dependent facilitation of the TC-GC axodendritic synapses in the IPL suggests roles of CCKergic TCs in regulating the odor-evoked $\gamma$ oscillations in the OB, a physiological behavior that depends on superficial GC-involved networks (Fourcaud-Trocmé et al., 2014) and is enhanced by high-frequency sniffing (Rosero and Aylwin, 2011) and critical for olfactory discrimination (Kay, 2014).

\section{References}

Antal M, Eyre M, Finklea B, Nusser Z (2006) External tufted cells in the main olfactory bulb form two distinct subpopulations. Eur J Neurosci 24:11241136.

Aroniadou-Anderjaska V, Zhou FM, Priest CA, Ennis M, Shipley MT (2000) Tonic and synaptically evoked presynaptic inhibition of sensory input to the rat olfactory bulb via GABA(B) heteroreceptors. J Neurophysiol 84:1194-1203.

Aungst JL, Heyward PM, Puche AC, Karnup SV, Hayar A, Szabo G, Shipley MT (2003) Centre-surround inhibition among olfactory bulb glomeruli. Nature 426:623-629.

Balu R, Pressler RT, Strowbridge BW (2007) Multiple modes of synaptic excitation of olfactory bulb granule cells. J Neurosci 27:5621-5632.

Banerjee A, Marbach F, Anselmi F, Koh MS, Davis MB, Garcia da Silva P, Delevich K, Oyibo HK, Gupta P, Li B, Albeanu DF (2015) An interglomerular circuit gates glomerular output and implements gain control in the mouse olfactory bulb. Neuron 87:193-207.

Belluscio L, Lodovichi C, Feinstein P, Mombaerts P, Katz LC (2002) Odorant receptors instruct functional circuitry in the mouse olfactory bulb. Nature 419:296-300.

Borisovska M, Bensen AL, Chong G, Westbrook GL (2013) Distinct modes of dopamine and GABA release in a dual transmitter neuron. J Neurosci 33:1790-1796.

Burton SD (2017) Inhibitory circuits of the mammalian main olfactory bulb. J Neurophysiol 118:2034-2051.

Cajal SR (1911) Histologie du systeme nerveux de I'homme et des vertebres. Paris: Maloine.

Carey RM, Wachowiak M (2011) Effect of sniffing on the temporal structure of mitral/tufted cell output from the olfactory bulb. J Neurosci 31:1061510626.

Cavarretta F, Burton SD, Igarashi KM, Shepherd GM, Hines ML, Migliore M (2018) Parallel odor processing by mitral and middle tufted cells in the olfactory bulb. Sci Rep 8:7625. 
Charpak S, Mertz J, Beaurepaire E, Moreaux L, Delaney K (2001) Odorevoked calcium signals in dendrites of rat mitral cells. Proc Natl Acad Sci USA 98:1230-1234.

Cheetham CE, Grier BD, Belluscio L (2015) Bulk regional viral injection in neonatal mice enables structural and functional interrogation of defined neuronal populations throughout targeted brain areas. Front Neural Circuits 9:72.

Dao NC, Brockway DF, Crowley NA (2019) In vitro optogenetic characterization of neuropeptide release from prefrontal cortical somatostatin neurons. Neuroscience 419:1-4.

De Saint Jan D, Hirnet D, Westbrook GL, Charpak S (2009) External tufted cells drive the output of olfactory bulb glomeruli. J Neurosci 29:20432052.

Doyle MW, Andresen MC (2001) Reliability of monosynaptic sensory transmission in brain stem neurons in vitro. J Neurophysiol 85:2213-2223.

Economo MN, Hansen KR, Wachowiak M (2016) Control of mitral/tufted cell output by selective inhibition among olfactory bulb glomeruli. Neuron 91:397-411.

Ennis M, Zhou FM, Ciombor KJ, Aroniadou-Anderjaska V, Hayar A, Borrelli E, Zimmer LA, Margolis F, Shipley MT (2001) Dopamine D2 receptor-mediated presynaptic inhibition of olfactory nerve terminals. J Neurophysiol 86:2986-2997.

Fourcaud-Trocmé N, Courtiol E, Buonviso N (2014) Two distinct olfactory bulb sublaminar networks involved in gamma and beta oscillation generation: a CSD study in the anesthetized rat. Front Neural Circuits 8:88.

Freeman WJ, Viana Di Prisco G, Davis GW, Whitney TM (1983) Conditioning of relative frequency of sniffing by rabbits to odors. J Comp Psychol 97:12-23.

Fukunaga I, Berning M, Kollo M, Schmaltz A, Schaefer AT (2012) Two distinct channels of olfactory bulb output. Neuron 75:320-329.

Fukunaga I, Herb JT, Kollo M, Boyden ES, Schaefer AT (2014) Independent control of gamma and theta activity by distinct interneuron networks in the olfactory bulb. Nat Neurosci 17:1208-1216.

Gil Z, Connors BW, Amitai Y (1999) Efficacy of thalamocortical and intracortical synaptic connections: quanta, innervation, and reliability. Neuron 23:385-397.

Gire DH, Schoppa NE (2009) Control of on/off glomerular signaling by a local GABAergic microcircuit in the olfactory bulb. J Neurosci 29:1345413464.

Haberly LB, Price JL (1977) The axonal projection patterns of the mitral and tufted cells of the olfactory bulb in the rat. Brain Res 129:152-157.

Hayar A, Karnup S, Shipley MT, Ennis M (2004a) Olfactory bulb glomeruli: external tufted cells intrinsically burst at theta frequency and are entrained by patterned olfactory input. J Neurosci 24:1190-1199.

Hayar A, Karnup S, Ennis M, Shipley MT (2004b) External tufted cells: a major excitatory element that coordinates glomerular activity. J Neurosci 24:6676-6685

Hughes J, Boden P, Costall B, Domeney A, Kelly E, Horwell DC, Hunter JC, Pinnock RD, Woodruff GN (1990) Development of a class of selective cholecystokinin type B receptor antagonists having potent anxiolytic activity. Proc Natl Acad Sci USA 87:6728-6732.

Igarashi KM, Ieki N, An M, Yamaguchi Y, Nagayama S, Kobayakawa K, Kobayakawa R, Tanifuji M, Sakano H, Chen WR, Mori K (2012) Parallel mitral and tufted cell pathways route distinct odor information to different targets in the olfactory cortex. J Neurosci 32:7970-7985.

Jackowski A, Parnavelas JG, Lieberman AR (1978) The reciprocal synapse in the external plexiform layer of the mammalian olfactory bulb. Brain Res 159:17-28.

Kay LM (2014) Circuit oscillations in odor perception and memory. Prog Brain Res 208:223-251.

Kishi K, Mori K, Ojima H (1984) Distribution of local axon collaterals of mitral, displaced mitral, and tufted cells in the rabbit olfactory bulb. J Comp Neurol 225:511-526.

Kiyokage E, Pan YZ, Shao Z, Kobayashi K, Szabo G, Yanagawa Y, Obata K, Okano H, Toida K, Puche AC, Shipley MT (2010) Molecular identity of periglomerular and short axon cells. J Neurosci 30:1185-1196.

Lin JY, Lin MZ, Steinbach P, Tsien RY (2009) Characterization of engineered channelrhodopsin variants with improved properties and kinetics. Biophys J 96:1803-1814.

Liu S, Shipley MT (2008a) Intrinsic conductances actively shape excitatory and inhibitory postsynaptic responses in olfactory bulb external tufted cells. J Neurosci 28:10311-10322.
Liu S, Shipley MT (2008b) Multiple conductances cooperatively regulate spontaneous bursting in mouse olfactory bulb external tufted cells. J Neurosci 28:1625-1639.

Liu S, Aungst JL, Puche AC, Shipley MT (2012) Serotonin modulates the population activity profile of olfactory bulb external tufted cells. J Neurophysiol 107:473-483.

Liu S, Plachez C, Shao Z, Puche A, Shipley MT (2013) Olfactory bulb short axon cell release of GABA and dopamine produces a temporally biphasic inhibition-excitation response in external tufted cells. J Neurosci 33:2916-2926.

Liu S, Puche AC, Shipley MT (2016) The interglomerular circuit potently inhibits olfactory bulb output neurons by both direct and indirect pathways. J Neurosci 36:9604-9617.

Liu WL, Shipley MT (1994) Intrabulbar associational system in the rat olfactory bulb comprises cholecystokinin-containing tufted cells that synapse onto the dendrites of GABAergic granule cells. J Comp Neurol 346:541558.

Liu X, Liu S (2018) Cholecystokinin selectively activates short axon cells to enhance inhibition of olfactory bulb output neurons. J Physiol 596:21852207.

Lledo PM, Gheusi G, Vincent JD (2005) Information processing in the mammalian olfactory system. Physiol Rev 85:281-317.

Lodovichi C, Belluscio L, Katz LC (2003) Functional topography of connections linking mirror-symmetric maps in the mouse olfactory bulb. Neuron 38:265-276.

López-Bendito G, Sturgess K, Erdélyi F, Szabó G, Molnár Z, Paulsen O (2004) Preferential origin and layer destination of GAD65-GFP cortical interneurons. Cereb Cortex 14:1122-1133.

Macrides F (1975) Temporal relationships between hippocampal slow waves and exploratory sniffing in hamsters. Behav Biol 14:295-308.

Macrides F, Chorover SL (1972) Olfactory bulb units: activity correlated with inhalation cycles and odor quality. Science 175:84-87.

Macrides F, Schneider SP (1982) Laminar organization of mitral and tufted cells in the main olfactory bulb of the adult hamster. J Comp Neurol 208:419-430.

Maher BJ, Westbrook GL (2008) Co-transmission of dopamine and GABA in periglomerular cells. J Neurophysiol 99:1559-1564.

Margrie TW, Schaefer AT (2003) Theta oscillation coupled spike latencies yield computational vigour in a mammalian sensory system. J Physiol 546:363-374.

Margrie TW, Sakmann B, Urban NN (2001) Action potential propagation in mitral cell lateral dendrites is decremental and controls recurrent and lateral inhibition in the mammalian olfactory bulb. Proc Natl Acad Sci USA 98:319-324.

Marks CA, Cheng K, Cummings DM, Belluscio L (2006) Activity-dependent plasticity in the olfactory intrabulbar map. J Neurosci 26:11257-11266.

Matsushita N, Okada H, Yasoshima Y, Takahashi K, Kiuchi K, Kobayashi K (2002) Dynamics of tyrosine hydroxylase promoter activity during midbrain dopaminergic neuron development. J Neurochem 82:295-304.

McGann JP (2013) Presynaptic inhibition of olfactory sensory neurons: new mechanisms and potential functions. Chem Senses 38:459-474.

Miles R (1986) Frequency dependence of synaptic transmission in nucleus of the solitary tract in vitro. J Neurophysiol 55:1076-1090.

Mombaerts P, Wang F, Dulac C, Chao SK, Nemes A, Mendelsohn M, Edmondson J, Axel R (1996) Visualizing an olfactory sensory map. Cell 87:675-686

Mori K, Kishi K, Ojima H (1983) Distribution of dendrites of mitral, displaced mitral, tufted, and granule cells in the rabbit olfactory bulb. J Comp Neurol 219:339-355.

Murphy GJ, Glickfeld LL, Balsen Z, Isaacson JS (2004) Sensory neuron signaling to the brain: properties of transmitter release from olfactory nerve terminals. J Neurosci 24:3023-3030.

Murphy GJ, Darcy DP, Isaacson JS (2005) Intraglomerular inhibition: signaling mechanisms of an olfactory microcircuit. Nat Neurosci 8:354-364.

Nagayama S, Enerva A, Fletcher ML, Masurkar AV, Igarashi KM, Mori K, Chen WR (2010) Differential axonal projection of mitral and tufted cells in the mouse main olfactory system. Front Neural Circuits 4:120.

Najac M, Sanz Diez A, Kumar A, Benito N, Charpak S, De Saint Jan D (2015) Intraglomerular lateral inhibition promotes spike timing variability in principal neurons of the olfactory bulb. J Neurosci 35:4319-4331 
Nickell WT, Behbehani MM, Shipley MT (1994) Evidence for GABAB-mediated inhibition of transmission from the olfactory nerve to mitral cells in the rat olfactory bulb. Brain Res Bull 35:119-123.

Orona E, Scott JW, Rainer EC (1983) Different granule cell populations innervate superficial and deep regions of the external plexiform layer in rat olfactory bulb. J Comp Neurol 217:227-237.

Orona E, Rainer EC, Scott JW (1984) Dendritic and axonal organization of mitral and tufted cells in the rat olfactory bulb. J Comp Neurol 226:346356.

Parrish-Aungst S, Shipley MT, Erdelyi F, Szabo G, Puche AC (2007) Quantitative analysis of neuronal diversity in the mouse olfactory bulb. J Comp Neurol 501:825-836.

Pinching AJ, Powell TP (1971) The neuron types of the glomerular layer of the olfactory bulb. J Cell Sci 9:305-345.

Pírez N, Wachowiak M (2008) In vivo modulation of sensory input to the olfactory bulb by tonic and activity-dependent presynaptic inhibition of receptor neurons. J Neurosci 28:6360-6371.

Price JL, Powell TP (1970a) The mitral and short axon cells of the olfactory bulb. J Cell Sci 7:631-651.

Price JL, Powell TP (1970b) The synaptology of the granule cells of the olfactory bulb. J Cell Sci 7:125-155.

Rall W, Shepherd GM, Reese TS, Brightman MW (1966) Dendrodendritic synaptic pathway for inhibition in the olfactory bulb. Exp Neurol 14:4456.

Ressler KJ, Sullivan SL, Buck LB (1994) Information coding in the olfactory system: evidence for a stereotyped and highly organized epitope map in the olfactory bulb. Cell 79:1245-1255.

Rosero MA, Aylwin ML (2011) Sniffing shapes the dynamics of olfactory bulb gamma oscillations in awake behaving rats. Eur J Neurosci 34:787799.

Sabatini BL, Regehr WG (1999) Timing of synaptic transmission. Annu Rev Physiol 61:521-542.

Schoenfeld TA, Marchand JE, Macrides F (1985) Topographic organization of tufted cell axonal projections in the hamster main olfactory bulb: an intrabulbar associational system. J Comp Neurol 235:503-518.

Schroeder CE, Wilson DA, Radman T, Scharfman H, Lakatos P (2010) Dynamics of active sensing and perceptual selection. Curr Opin Neurobiol 20:172-176.

Seroogy KB, Brecha N, Gall C (1985) Distribution of cholecystokinin-like immunoreactivity in the rat main olfactory bulb. J Comp Neurol 239:373383.

Shao Z, Puche AC, Kiyokage E, Szabo G, Shipley MT (2009) Two GABAergic intraglomerular circuits differentially regulate tonic and phasic presynaptic inhibition of olfactory nerve terminals. J Neurophysiol 101:1988-2001.

Shao Z, Puche AC, Liu S, Shipley MT (2012) Intraglomerular inhibition shapes the strength and temporal structure of glomerular output. J Neurophysiol 108:782-793.
Shepherd GM (1972) Synaptic organization of the mammalian olfactory bulb. Physiol Rev 52:864-917.

Shipley MT, Ennis M (1996) Functional organization of olfactory system. J Neurobiol 30:123-176

Short SM, Wachowiak M (2019) Temporal dynamics of inhalation-linked activity across defined subpopulations of mouse olfactory bulb neurons imaged in vivo. eNeuro 6: ENEURO.0189-19.2019.

Tsai HC, Zhang F, Adamantidis A, Stuber GD, Bonci A, de Lecea L, Deisseroth K (2009) Phasic firing in dopaminergic neurons is sufficient for behavioral conditioning. Science 324:1080-1084.

Vaaga CE, Westbrook GL (2017) Distinct temporal filters in mitral cells and external tufted cells of the olfactory bulb. J Physiol 595:6349-6362.

van den Pol AN (2012) Neuropeptide transmission in brain circuits. Neuron 76:98-115.

Vassar R, Chao SK, Sitcheran R, Nuñez JM, Vosshall LB, Axel R (1994) Topographic organization of sensory projections to the olfactory bulb. Cell 79:981-991.

Verhagen JV, Wesson DW, Netoff TI, White JA, Wachowiak M (2007) Sniffing controls an adaptive filter of sensory input to the olfactory bulb. Nat Neurosci 10:631-639.

Wachowiak M (2011) All in a sniff: olfaction as a model for active sensing. Neuron 71:962-973.

Wachowiak M, Cohen LB (1999) Presynaptic inhibition of primary olfactory afferents mediated by different mechanisms in lobster and turtle. J Neurosci 19:8808-8817.

Watakabe A, Ohtsuka M, Kinoshita M, Takaji M, Isa K, Mizukami H, Ozawa K, Isa T, Yamamori T (2015) Comparative analyses of adeno-associated viral vector serotypes $1,2,5,8$ and 9 in marmoset, mouse and macaque cerebral cortex. Neurosci Res 93:144-157.

Welker WI (1964) Analysis of sniffing of the albino rat. Behaviour 22:223244.

Wellis DP, Scott JW (1990) Intracellular responses of identified rat olfactory bulb interneurons to electrical and odor stimulation. J Neurophysiol 64:932-947.

Whitesell JD, Sorensen KA, Jarvie BC, Hentges ST, Schoppa NE (2013) Interglomerular lateral inhibition targeted on external tufted cells in the olfactory bulb. J Neurosci 33:1552-1563.

Yoshimura M, Jessell TM (1989) Primary afferent-evoked synaptic responses and slow potential generation in rat substantia gelatinosa neurons in vitro. J Neurophysiol 62:96-108.

Youngentob SL, Mozell MM, Sheehe PR, Hornung DE (1987) A quantitative analysis of sniffing strategies in rats performing odor detection tasks. Physiol Behav 41:59-69.

Zingg B, Chou XL, Zhang ZG, Mesik L, Liang F, Tao HW, Zhang LI (2017) AAV-mediated anterograde transsynaptic tagging: mapping corticocollicular input-defined neural pathways for defense behaviors. Neuron 93:33-47. 